\title{
On the Multilinear Singular Integrals and Commutators in the Weighted Amalgam Spaces
}

\author{
Feng Liu, Huoxiong Wu, and Daiqing Zhang \\ School of Mathematical Sciences, Xiamen University, Xiamen, Fujian 361005, China \\ Correspondence should be addressed to Daiqing Zhang; zhangdaiqing2011@163.com \\ Received 28 September 2013; Revised 5 January 2014; Accepted 5 January 2014; Published 4 May 2014 \\ Academic Editor: Yoshihiro Sawano
}

Copyright ( 2014 Feng Liu et al. This is an open access article distributed under the Creative Commons Attribution License, which permits unrestricted use, distribution, and reproduction in any medium, provided the original work is properly cited.

This paper is concerned with the norm estimates for the multilinear singular integral operators and their commutators formed by BMO functions on the weighted amalgam spaces $\left(L_{v_{\vec{w}}}^{q}, L^{p}\right)^{\alpha}\left(\mathbb{R}^{n}\right)$. Some criterions of boundedness for such operators in $\left(L_{v_{\vec{w}}}^{q}, L^{p}\right)^{\alpha}\left(\mathbb{R}^{n}\right)$ are given. As applications, the norm inequalities for the multilinear Calderón-Zygmund operators and multilinear singular integrals with nonsmooth kernels as well as the corresponding commutators on $\left(L_{v_{\vec{w}}}^{q}, L^{p}\right)^{\alpha}\left(\mathbb{R}^{n}\right)$ are obtained.

\section{Introduction}

Let $\mathbb{R}^{n}(n \geq 2)$ be the $n$-dimensional Euclidean space equipped with the Euclidean norm $|\cdot|$ and the Lebesgue measure $d x$. For $1 \leq p, q \leq \infty$; the amalgam spaces $\left(L^{q}, L^{p}\right)\left(\mathbb{R}^{n}\right)$ of $L^{p}\left(\mathbb{R}^{n}\right)$ and $L^{q}\left(\mathbb{R}^{n}\right)$ are denoted by the set of all measurable functions $f: \mathbb{R}^{n} \rightarrow \mathbb{C}$, which are locally in $L^{q}\left(\mathbb{R}^{n}\right)$ and satisfy

$$
\|f\|_{\left(L^{q}, L^{p}\right)\left(\mathbb{R}^{n}\right)}:=\left(\int_{\mathbb{R}^{n}}\left\|f \chi_{B(y, 1)}\right\|_{q}^{p} d y\right)^{1 / p}<\infty,
$$

where $B(y, r):=\left\{x \in \mathbb{R}^{n}:|x-y|<r\right\}$ for $r>0$ and $y \in \mathbb{R}^{n}$. We remark that the amalgam spaces $\left(L^{q}, L^{p}\right)\left(\mathbb{R}^{n}\right)$ were introduced by Fofana in [1] in connection with the study of the continuity of the fractional maximal operator of Hardy-Littlewood and of the Fourier transformation in $\mathbb{R}^{n}$. In [1], Fofana also considered the subspace $\left(L^{q}, L^{p}\right)^{\alpha}\left(\mathbb{R}^{n}\right)$ of $\left(L^{q}, L^{p}\right)\left(\mathbb{R}^{n}\right)$, which consists of measurable functions $f$ such that for $1 \leq \alpha \leq \infty$,

$$
\begin{aligned}
& \|f\|_{\left(L^{q}, L^{p}\right)^{\alpha}\left(\mathbb{R}^{n}\right)} \\
& \quad:=\sup _{r>0}\left(\int_{\mathbb{R}^{n}}\left(|B(y, r)|^{1 / \alpha-1 / q-1 / p}\left\|f \chi_{B(y, r)}\right\|_{L^{q}\left(\mathbb{R}^{n}\right)}\right)^{p} d y\right)^{1 / p} \\
& \quad<\infty, \quad 1 \leq p, q<\infty
\end{aligned}
$$

By the definitions, it is clear (also see [1]) that $\left(L^{q}\right.$, $\left.L^{q}\right)\left(\mathbb{R}^{n}\right)=L^{q}\left(\mathbb{R}^{n}\right),\left(L^{q}, L^{\infty}\right)^{\alpha}\left(\mathbb{R}^{n}\right)=L^{q,(n q / \alpha)}\left(\mathbb{R}^{n}\right)$, where $L^{q, \lambda}\left(\mathbb{R}^{n}\right)$, with $1 \leq q<\infty$ and $0<\lambda<n$, is the classical Morrey space that consists of measurable functions $f: \mathbb{R}^{n} \rightarrow$ $\mathbb{C}$ such that

$$
\begin{aligned}
& \|f\|_{L^{q, \lambda}\left(\mathbb{R}^{n}\right)} \\
& \quad:=\left(\sup _{y \in \mathbb{R}^{n}, r>0}|B(y, r)|^{\lambda / n-1} \int_{B(y, r)}|f(x)|^{q} d x\right)^{1 / q}<\infty .
\end{aligned}
$$

In this paper, we focus on the weighted version of $\left(L^{q}, L^{p}\right)^{\alpha}\left(\mathbb{R}^{n}\right)$. Precisely, letting $w$ be a weight on $\mathbb{R}^{n}$ and $1 \leq q, p, \alpha \leq \infty$, we define the weighted amalgam spaces $\left(L_{w}^{q}, L^{p}\right)^{\alpha}\left(\mathbb{R}^{n}\right)$ as the space of all measurable functions $f$ satisfying

$$
\begin{array}{r}
\|f\|_{\left(L_{w}^{q}, L^{p}\right)^{\alpha}\left(\mathbb{R}^{n}\right)} \\
:=\sup _{r>0}\left(\int _ { \mathbb { R } ^ { n } } \left(w(B(y, r))^{1 / \alpha-1 / q-1 / p}\right.\right. \\
\left.\left.\quad \times\left\|f \chi_{B(y, r)}\right\|_{L_{w}^{q}\left(\mathbb{R}^{n}\right)}\right)^{p} d y\right)^{1 / p}
\end{array}
$$

and a suitable modification version for $p=\infty$ or $q=\infty$.

$$
<\infty, \quad 1 \leq p, q<\infty
$$


and a suitable modification version for $p=\infty$ or $q=\infty$, where $L_{w}^{q}\left(\mathbb{R}^{n}\right)$ is the weighted Lebesgue space.

It is easy to check that when $\kappa=1-q / \alpha$ and $1 \leq q<$ $\alpha<\infty$, the space $\left(L_{w}^{q}, L^{\infty}\right)^{\alpha}\left(\mathbb{R}^{n}\right)$ is nothing but the weighted Morrey space $L_{w}^{q, \kappa}\left(\mathbb{R}^{n}\right)$, which is the set of all measurable functions $f$ such that (see [2])

$$
\begin{aligned}
& \|f\|_{L_{w}^{q, \kappa}\left(\mathbb{R}^{n}\right)} \\
& \quad:=\sup _{B}\left(\frac{1}{w(B)^{\kappa}} \int_{B}|f(x)|^{q} w(x) d x\right)^{1 / q}<\infty, \\
& 1 \leq q<\infty, \quad 0<\kappa<1 .
\end{aligned}
$$

As is well known, the boundedness of the classical operators in the harmonic analysis on the weighted Morrey spaces has extensively been studied (see [2-6] and references therein). In particular, Wang and Yi [6] recently showed that the $m$-linear commutators and the iterated commutators of the $m$-linear Calderón-Zygmund operators are bounded on weighted Morrey spaces.

Based on the above, we feel that it is natural and interesting to study the boundedness of the classical operators in harmonic analysis on the amalgam spaces and the weighted versions. Indeed, a lot of attention has recently been given to this topic (e.g., see [7-10]). Here, we will continue the investigation along this line. The main purpose of this paper is to study the boundedness of the multilinear operators on the weighted amalgam spaces $\left(L_{v_{\vec{w}}}^{q}, L^{p}\right)^{\alpha}\left(\mathbb{R}^{n}\right)$.

Let $K\left(x, y_{1}, \ldots, y_{m}\right)$ be a locally integral function defined off the diagonal $x=y_{1}=\cdots=y_{m}$ in $\left(\mathbb{R}^{n}\right)^{m+1}$ and let $T$ : $\mathcal{S}\left(\mathbb{R}^{n}\right) \times \cdots \times \mathcal{S}\left(\mathbb{R}^{n}\right) \rightarrow \mathcal{S}^{\prime}\left(\mathbb{R}^{n}\right)$ be an $m$-linear operator associated with the kernel $K\left(x, y_{1}, \ldots, y_{m}\right)$ in the following way:

$$
\begin{aligned}
& \left\langle T\left(f_{1}, \ldots, f_{m}\right), g\right\rangle \\
& =\int_{\mathbb{R}^{n}} \int_{\left(\mathbb{R}^{n}\right)^{m}} K\left(x, y_{1}, \ldots, y_{m}\right) \prod_{i=1}^{m} f_{i}\left(y_{i}\right) g(x) d y_{1} \cdots d y_{m} d x,
\end{aligned}
$$

where $f_{1}, \ldots, f_{m}, g$ in $\mathcal{S}\left(\mathbb{R}^{n}\right)$ with $\bigcap_{j=1}^{m} \operatorname{supp}\left(f_{j}\right) \bigcap \operatorname{supp}(g)=$ $\emptyset$.

For $\vec{b}=\left(b_{1}, \ldots, b_{m}\right) \in\left(\operatorname{BMO}\left(\mathbb{R}^{n}\right)\right)^{m}$, we define the $m$ linear commutator of $T$ denoted by $T_{\Sigma \vec{b}}$ as follows:

$$
T_{\Sigma \vec{b}}\left(f_{1}, \ldots, f_{m}\right):=\sum_{j=1}^{m} T_{\vec{b}}^{j}(\vec{f}),
$$

where each term is the commutator of $b_{j}$ and $T$ in the $j$ th entry of $T$; that is

$$
T_{\vec{b}}^{j}(\vec{f}):=b_{j} T\left(f_{1}, \ldots, f_{m}\right)-T\left(f_{1}, \ldots, b_{j} f_{j}, \ldots, f_{m}\right)
$$

and $\vec{f}=\left(f_{1}, \ldots, f_{m}\right)$, where $f_{j}$ is a smooth function with compact support on $\mathbb{R}^{n}$. The iterated commutator $T_{\Pi \vec{b}}$ is defined by

$$
T_{\Pi \vec{b}}(\vec{f}):=\left[b_{1},\left[b_{2}, \ldots,\left[b_{m-1},\left[b_{m}, T\right]_{m}\right]_{m-1}, \ldots\right]_{2}\right]_{1}(\vec{f}) .
$$

If $T$ is associated with a distribution kernel, which coincides with the above function $K$, then we have, at a formal level,

$$
\begin{aligned}
& T(\vec{f})(x) \\
& =\int_{\left(\mathbb{R}^{n}\right)^{m}} K\left(x, y_{1}, \ldots, y_{m}\right) f_{1}\left(y_{1}\right) \cdots f_{m}\left(y_{m}\right) d y_{1} \cdots d y_{m} ; \\
& T_{\vec{b}}^{j}(\vec{f})(x) \\
& =\int_{\left(\mathbb{R}^{n}\right)^{m}}\left(b_{j}(x)-b_{j}\left(y_{j}\right)\right) \\
& T_{\Pi \vec{b}}(\vec{f})(x) \\
& =\int_{\left(\mathbb{R}^{n}\right)^{m}} \prod_{j=1}^{m}\left(b_{j}(x)-b_{j}\left(y_{j}\right)\right) K\left(x, y_{1}, \ldots, y_{m}\right) f_{1}\left(y_{1}\right) \cdots f_{m}\left(y_{m}\right) d y_{1} \cdots d y_{m}, \\
& \times f_{1}\left(y_{1}\right) \cdots f_{m}\left(y_{m}\right) d y_{1} \cdots d y_{m} .
\end{aligned}
$$

Also, we recall the definitions of the classical Muckenhoupt classes $A_{p}$ weights and the multilinear $A_{\vec{P}}$ conditions for multiple weights.

Definition 1. A weighted $w$ on $\mathbb{R}^{n}$, that is, a positive locally integrable function on $\mathbb{R}^{n}$, belongs to $A_{p}\left(\mathbb{R}^{n}\right)$ for $1<p<\infty$ if there exists a constant $C>0$ such that

$$
\begin{aligned}
\sup _{Q \text { cube in } \mathbb{R}^{n}}\left(\frac{1}{|Q|} \int_{Q} w(x) d x\right) \\
\quad \times\left(\frac{1}{|Q|} \int_{Q} w^{-1 /(p-1)}(x) d x\right)^{p-1} \leq C .
\end{aligned}
$$

The infimum of these constants $C$ is called the $A_{p}$ constant of $w$ and denoted by $[w]_{A_{p}}$. A weight $w$ belongs to the class $A_{1}\left(\mathbb{R}^{n}\right)$ if there exists a constant $C>0$ such that

$$
\sup _{Q \text { cube in } \mathbb{R}^{n}} \frac{1}{|Q|} \int_{Q} w(x) d x\left(\inf _{x \in Q} w(x)\right)^{-1} \leq C,
$$

and the infimum of these constants $C$ is called the $A_{1}$ constant of $w$ and is denoted by $[w]_{A_{1}}$.

Definition 2. Let $m \in \mathbb{N}$ with $m \geq 1$ and $1 \leq p_{1}, \ldots, p_{m}<\infty$, $1 / m \leq p<\infty$, and $1 / p=\sum_{i=1}^{m} 1 / p_{i}$. Let $\vec{P}=\left(p_{1}, \ldots, p_{m}\right)$ and $\vec{w}=\left(w_{1}, \ldots, w_{m}\right)$. Set

$$
v_{\vec{w}}=\prod_{j=1}^{m} w_{j}^{p / p_{j}}
$$


We say that $\vec{w}$ satisfies the $A_{\vec{P}}$ condition if

$$
\begin{aligned}
& \sup _{Q}\left(\frac{1}{|Q|} \int_{Q} v_{\vec{w}}(x) d x\right)^{1 / p} \\
& \quad \times \prod_{j=1}^{m}\left(\frac{1}{|Q|} \int_{Q} w_{j}^{1-p_{j}^{\prime}}(x) d x\right)^{1 / p_{j}^{\prime}}<\infty,
\end{aligned}
$$

where $p_{j}^{\prime}=p_{j} /\left(p_{j}-1\right)$ for $j=1, \ldots, m$.

Obviously, for $m=1, A_{\vec{P}}$ is the classical Muckenhoupt classes $A_{p}$ condition. It is not difficult to check that for $m>1$ (see $[11])$,

$$
\prod_{j=1}^{m} A_{p_{j}} \subsetneq A_{\vec{P}}
$$

which implies that something more general happens for the $A_{\vec{P}}$ classes. Also, the authors in [11] showed that the $A_{\vec{P}}$ conditions are the largest classes of weights because all $\mathrm{m}$ linear Calderón-Zygmund operators are bounded on the weighted Lebesgue spaces.

To state our main results, we still need to recall and introduce some notations. For fixed $y \in \mathbb{R}^{n}$ and $r>0$, we set $B=B(y, r)$. For any $\lambda>0$, let $\lambda B=B(y, \lambda r)$ and $\chi_{\lambda B}$ be the characteristic function of the set $\lambda B$. Given any positive integer $m$ and $j \in\{1, \ldots, m\}$, we denote by $D_{j}^{m}$ the family of all finite subset $\sigma=\{\sigma(1), \ldots, \sigma(j)\}$ of $\{1, \ldots, m\}$ of $j$ different elements. For any $\sigma \in D_{j}^{m}$, we also denote the complementary sequence of $\sigma$ by $\tau$ given by $\tau=\{1, \ldots, m\} \backslash \sigma$. We remark that $\tau=\emptyset$ if and only if $\sigma \in D_{m}^{m}$. Letting $\vec{f}=\left(f_{1}, \ldots, f_{m}\right)$ for a fixed $j \in\{0, \ldots, m\}$ and $\sigma \in D_{j}^{m}$, we set $\overrightarrow{f^{\sigma}}=\left(\tilde{f}_{1}, \ldots, \tilde{f}_{m}\right)$ and $\tilde{f}_{i}=f_{i} \chi_{(2 B)^{c}}$ if $i \in \sigma$ and $\tilde{f}_{i}=f_{i} \chi_{2 B}$ if $i \in \tau$. Now we can formulate our main results as follows.

Theorem 3. Let $m \in \mathbb{N}$ with $m \geq 2$ and $T$ be an $m$-linear operator. Let $1<q_{j} \leq \alpha_{j}<p_{j}<\infty(j=1, \ldots, m)$ satisfy $1 / q=\sum_{i=1}^{m} 1 / q_{i}, 1 / \alpha=\sum_{i=1}^{m} 1 / \alpha_{i}, 1 / p=\sum_{i=1}^{m} 1 / p_{i}$, and $p / p_{j}=q / q_{j}=\alpha / \alpha_{j}(j=1, \ldots, m)$. Assume that $\vec{w}=$ $\left(w_{1}, \ldots, w_{m}\right) \in A_{\vec{Q}}$ for $\vec{Q}=\left(q_{1}, \ldots, q_{m}\right)$ with $w_{1}, \ldots, w_{m} \in$ $A_{\infty}$ and $v_{\vec{w}}=\prod_{j=1}^{m} w_{j}^{q / q_{j}}$. If T maps $L_{w_{1}}^{q_{1}}\left(\mathbb{R}^{n}\right) \times \cdots \times L_{w_{m}}^{q_{m}}\left(\mathbb{R}^{n}\right)$ to $L_{v_{\vec{w}}}^{q}\left(\mathbb{R}^{n}\right)$, then the inequality

$$
\|T(\vec{f})\|_{\left(L_{v_{\vec{w}}}^{q}, L^{p}\right)^{\alpha}\left(\mathbb{R}^{n}\right)} \leq C \prod_{j=1}^{m}\left\|f_{j}\right\|_{\left(L_{w_{j}}, L^{q_{j}}\right)^{\alpha_{j}}\left(\mathbb{R}^{n}\right)}
$$

holds provided that for any ball $B$ in $\mathbb{R}^{n}$, any $j \in\{1, \ldots, m\}$ and $\sigma \in D_{j}^{m}$, there exist constants $\gamma \geq 0$ and $\beta \geq 0$ such that for a.e. $x \in B$,

$$
\begin{aligned}
\left|T\left(\overrightarrow{f^{\sigma}}\right)(x)\right| \\
\leq C\left(\prod_{i \in \mathcal{\tau}} w_{i}(2 B)^{-1 / q_{i}}\left\|f_{i} \chi_{2 B}\right\|_{L_{w_{i}}^{q_{i}}}\right) \\
\quad \times \prod_{i \in \sigma k=1}^{\infty} \frac{1+\gamma k}{2^{k \beta}} w_{i}\left(2^{k+1} B\right)^{-1 / q_{i}}\left\|f_{i} \chi_{2^{k+1} B}\right\|_{L_{w_{i}}^{q_{i}}} .
\end{aligned}
$$

Theorem 4. Let $m \in \mathbb{N}$ with $m \geq 2,1<q_{j} \leq \alpha_{j}<$ $p_{j}<\infty(j=1, \ldots, m)$, satisfy $1 / q=\sum_{i=1}^{m} 1 / q_{i}, 1 / \alpha=$ $\sum_{i=1}^{m} 1 / \alpha_{i}, 1 / p=\sum_{i=1}^{m} 1 / p_{i}$, and $p / p_{j}=q / q_{j}=\alpha / \alpha_{j}(j=$ $1, \ldots, m)$. Assume that $\vec{w}=\left(w_{1}, \ldots, w_{m}\right) \in A_{\vec{Q}}$ for $\vec{Q}=$ $\left(q_{1}, \ldots, q_{m}\right)$ with $w_{1}, \ldots, w_{m} \in A_{\infty}, v_{\vec{w}}=\prod_{j=1}^{m} w_{j}^{q / q_{j}}$, and $\vec{b}=\left(b_{1}, \ldots, b_{m}\right) \in B M O^{m}$. If

$$
\begin{aligned}
& \left\|T_{\Sigma \vec{b}}(\vec{f})\right\|_{L_{v_{\vec{w}}}^{q}\left(\mathbb{R}^{n}\right)} \\
& \quad \leq C\left(\sum_{j=1}^{m}\left\|b_{j}\right\|_{B M O\left(\mathbb{R}^{n}\right)}\right) \prod_{j=1}^{m}\left\|f_{j}\right\|_{L_{w_{j}}^{q_{j}}\left(\mathbb{R}^{n}\right)^{\prime}}
\end{aligned}
$$

then the inequality

$$
\begin{aligned}
& \left\|T_{\Sigma \vec{b}}(\vec{f})\right\|_{\left(L_{v_{\vec{w}}}^{q}, L^{p}\right)^{\alpha}\left(\mathbb{R}^{n}\right)} \\
& \leq C\left(\sum_{j=1}^{m}\left\|b_{j}\right\|_{B M O\left(\mathbb{R}^{n}\right)}\right) \prod_{j=1}^{m}\left\|f_{j}\right\|_{\left(L_{w_{j}}^{q_{j}} L^{p_{j}}\right)^{\alpha_{j}}\left(\mathbb{R}^{n}\right)}
\end{aligned}
$$

holds provided that for any ball $B$ in $\mathbb{R}^{n}$, any $j \in\{1, \ldots, m\}$, $\sigma \in D_{j}^{m}, \mu \in \sigma$, and $\nu \in \tau$, there exist constants $\gamma \geq 0$ and $\beta \geq 0$ such that for a.e. $x \in B$,

$$
\begin{aligned}
& \left|T_{\vec{b}}^{\mu}\left(\overrightarrow{f^{\sigma}}\right)(x)\right| \leq C\left(\prod_{i \in \tau} w_{i}(2 B)^{-1 / q_{i}}\left\|f_{i} \chi_{2 B}\right\|_{L_{w_{i}}^{q_{i}}}\right) \\
& \times\left(\prod_{i \in \sigma \backslash\{\mu\}} \sum_{k=1}^{\infty} \frac{1+\gamma k}{2^{k \beta}} w_{i}\left(2^{k+1} B\right)^{-1 / q_{i}}\right. \\
& \left.\times\left\|f_{i} \chi_{2^{k+1} B}\right\|_{L_{w_{i}}^{q_{i}}}\right) \\
& \times \sum_{k=1}^{\infty}\left(\left|b_{\mu}(x)-\left(b_{\mu}\right)_{2^{k+1} B}\right|+k\left\|b_{\mu}\right\|_{B M O}\right) w_{\mu} \\
& \times\left(2^{k+1} B\right)^{-1 / q_{\mu}}\left\|f_{\mu} \chi_{2^{k+1} B}\right\|_{L_{w_{\mu}}^{q_{\mu}}}, \\
& \left|T_{\vec{b}}^{v}\left(\overrightarrow{f^{\sigma}}\right)(x)\right| \leq C\left(\prod_{i \in \tau \backslash\{v\}} w_{i}(2 B)^{-1 / q_{i}}\left\|f_{i} \chi_{2 B}\right\|_{L_{w_{i}}^{q_{i}}}\right) \\
& \times\left(\prod_{i \in \sigma} \sum_{k=1}^{\infty} \frac{1+\gamma k}{2^{k \beta}} w_{i}\left(2^{k+1} B\right)^{-1 / q_{i}}\right. \\
& \left.\times\left\|f_{i} \chi_{2^{k+1} B}\right\|_{L_{w_{i}}^{q_{i}}}\right) \\
& \times\left(\left|b_{v}(x)-\left(b_{v}\right)_{2 B}\right|+\left\|b_{\nu}\right\|_{B M O}\right) w_{\nu} \\
& \times(2 B)^{-1 / q_{\nu}}\left\|f_{\nu} \chi_{2 B}\right\|_{L_{w_{\nu}}^{q_{\nu}}} .
\end{aligned}
$$


Theorem 5. Let $m \in \mathbb{N}$ with $m \geq 2,1<q_{j} \leq \alpha_{j}<$ $p_{j}<\infty(j=1, \ldots, m)$, satisfy $1 / q=\sum_{i=1}^{m} 1 / q_{i}, 1 / \alpha=$ $\sum_{i=1}^{m} 1 / \alpha_{i}, 1 / p=\sum_{i=1}^{m} 1 / p_{i}$, and $p / p_{j}=q / q_{j}=\alpha / \alpha_{j}(j=$ $1, \ldots, m)$. Suppose that $\vec{w}=\left(w_{1}, \ldots, w_{m}\right) \in A_{\vec{Q}}$ for $\vec{Q}=$ $\left(q_{1}, \ldots, q_{m}\right)$ with $w_{1}, \ldots, w_{m} \in A_{\infty}, v_{\vec{w}}=\prod_{j=1}^{m} w_{j}^{q / q_{j}}$, and $\vec{b}=\left(b_{1}, \ldots, b_{m}\right) \in B M O^{m}$. If

$$
\begin{aligned}
& \left\|T_{\Pi \vec{b}}(\vec{f})\right\|_{L_{v_{\vec{w}}}^{q}\left(\mathbb{R}^{n}\right)} \\
& \quad \leq C\left(\prod_{j=1}^{m}\left\|b_{j}\right\|_{B M O\left(\mathbb{R}^{n}\right)}\right) \prod_{j=1}^{m}\left\|f_{j}\right\|_{L_{w_{j}}^{q_{j}}\left(\mathbb{R}^{n}\right)},
\end{aligned}
$$

then the inequality

$$
\begin{aligned}
& \left\|T_{\Pi \vec{b}}(\vec{f})\right\|_{\left(L_{\vec{v}}^{q}, L^{p}\right)^{\alpha}\left(\mathbb{R}^{n}\right)} \\
& \quad \leq C\left(\prod_{j=1}^{m}\left\|b_{j}\right\|_{B M O\left(\mathbb{R}^{n}\right)}\right) \prod_{j=1}^{m}\left\|f_{j}\right\|_{\left(L_{w_{j}}^{q_{j}}, L^{p_{j}}\right)^{\alpha_{j}}\left(\mathbb{R}^{n}\right)}
\end{aligned}
$$

holds provided that for any ball $B$ in $\mathbb{R}^{n}$, any $j \in\{1, \ldots, m\}$ and $\sigma \in D_{j}^{m}$, there exist constants $\gamma \geq 0$ and $\beta \geq 0$ such that for a.e. $x \in B$,

$$
\begin{aligned}
\left|T_{\Pi \vec{b}}\left(\overrightarrow{f^{\sigma}}\right)(x)\right| \leq & C \sum_{\eta=0}^{m} \sum_{\sigma_{0} \in D_{\eta}^{m}}\left(\prod_{i \in \sigma_{0}}\left|b_{i}(x)-\left(b_{i}\right)_{2 B}\right|\right) \\
& \times\left(\prod_{i \in \tau_{0}}\left\|b_{i}\right\|_{B M O}\right) \\
& \times\left(\prod_{i \in \tau} w_{i}(2 B)^{-1 / q_{i}}\left\|f_{i} \chi_{2 B}\right\|_{L_{w_{i}}^{q_{i}}}\right) \\
& \times \prod_{i \in \sigma} \sum_{k=1}^{\infty} \frac{1+\gamma k}{2^{k \beta}} w_{i}\left(2^{k+1} B\right)^{-1 / q_{i}}\left\|f_{i} \chi_{2^{k+1} B}\right\|_{L_{w_{i}}^{q_{i}}}
\end{aligned}
$$

where for any $\eta \in\{0,1, \ldots, m\}, \sigma_{0} \in D_{\eta}^{m}$ and $\tau_{0}=\{1, \ldots, m\} \backslash$ $\sigma_{0}$.

Theorem 6. Let $T$ be an m-linear operator with kernel $K$ satisfying

$$
\left|K\left(y_{0}, y_{1}, \ldots, y_{m}\right)\right| \leq \frac{A}{\left(\sum_{k, l=0}^{m}\left|y_{k}-y_{l}\right|\right)^{m n}} .
$$

Let $1<q_{j} \leq \alpha_{j}<p_{j}<\infty(j=1, \ldots, m)$ satisfy $1 / q=$ $\sum_{i=1}^{m} 1 / q_{i}, 1 / \alpha=\sum_{i=1}^{m} 1 / \alpha_{i}, 1 / p=\sum_{i=1}^{m} 1 / p_{i}$, and $p / p_{j}=$ $q / q_{j}=\alpha / \alpha_{j}(j=1, \ldots, m)$. Assume that $\vec{w}=\left(w_{1}, \ldots, w_{m}\right) \in$ $\prod_{j=1}^{m} A_{q_{j}}, v_{\vec{w}}=\prod_{j=1}^{m} w_{j}^{q / q_{j}}$, and $\vec{b}=\left(b_{1}, \ldots, b_{m}\right) \in B M O^{m}$. Then these inequalities (17), (20)-(21), and (24) hold.
Remark 7. We remark that for $p_{1}=\cdots=p_{m}=p=\infty$, Theorems 3-6 are also true, just with the restrictive condition: $q / q_{j}=\alpha / \alpha_{j}(j=1, \ldots, m)$. Moreover, for $v_{\vec{w}}=\prod_{j=1}^{m} w_{j}^{q / q_{j}}=$ $w \in A_{q}$, that is, $w_{1}=\cdots=w_{m}=w \in A_{q}$, we can remove the restrictive condition $p / p_{j}=q / q_{j}=\alpha / \alpha_{j}(j=1, \ldots, m)$ in Theorems 3-6. See also [12, Theorem 3.5] for the unweighted case.

The rest of this paper is organized as follows. In Section 2, we will give the proofs of our main results. Some applications will be given in Section 3. Throughout this paper, the letter $C$, sometimes with additional parameters, will stand for positive constants, not necessarily the same one at each occurrence but independent of the essential variables. In what follows, we use the convention $\prod_{j \in \emptyset} a_{j}=1$ and $\sum_{j \in \emptyset} a_{j}=0$.

\section{The Proofs of Main Results}

Let us begin with a lemma, which will be used in the proofs of our main results.

Lemma 8 (cf. [6, Lemma 3.1]). Let $m \geq 2, p_{1}, \ldots, p_{m} \in$ $(0, \infty)$ and $p \in(0, \infty)$ with $1 / p=\sum_{i=1}^{m} 1 / p_{i}$. Assume that $w_{1}, \ldots, w_{m} \in A_{\infty}$ and $v_{\vec{w}}=\prod_{i=1}^{m} w_{i}^{p / p_{i}}$, then for any ball $B$, there exists a constant $C>0$ such that

$$
\prod_{i=1}^{m}\left(\int_{B} w_{i}(x) d x\right)^{p / p_{i}} \leq C \int_{B} v_{\vec{w}}(x) d x .
$$

Proof of Theorem 3. For fixed $x \in B$, we can write

$$
T(\vec{f})(x)=T\left(\vec{f} \chi_{2 B}\right)(x)+\sum_{j=1}^{m} \sum_{\sigma \in D_{j}^{m}} T\left(\overrightarrow{f^{\sigma}}\right)(x) .
$$

The boundedness of $T$ from $L_{w_{1}}^{q_{1}} \times \cdots \times L_{w_{m}}^{q_{m}}$ to $L_{v_{\vec{w}}}^{q},(17)$ and Hölder's inequality lead to

$$
\begin{gathered}
\left\|T(\vec{f}) \chi_{B}\right\|_{L_{v_{\vec{w}}}^{q}} \\
\leq C \prod_{i=1}^{m}\left\|f_{i} \chi_{2 B}\right\|_{L_{w_{i}}^{q_{i}}} \\
+C \sum_{j=1}^{m} \sum_{\sigma \in D_{j}^{m}}\left(\prod_{i \in \tau}\left(\frac{w_{i}(B)}{w_{i}(2 B)}\right)^{1 / q_{i}}\right. \\
\left.\times \prod_{i \in \sigma} \sum_{k=1}^{\infty} \frac{1+\gamma k}{2^{k \beta}}\left(\frac{w_{i}(B)}{w_{i}\left(2^{k+1} B\right)}\right)^{1 / q_{i} B} \|_{L_{w_{i}}^{q_{i}}}\right) \\
\times\left\|f_{i} \chi_{2^{k+1} B}\right\|_{L_{w_{i}}^{q_{i}}}
\end{gathered}
$$

Note that for any $k \in \mathbb{N}$ and $w \in A_{q}$, there exists a constant $c_{q}>0$ that depends only on $q,[w]_{A_{q}}, n$ such that

$$
\frac{w(B)}{w\left(2^{k+1} B\right)} \leq C 2^{-(k+1) n c_{q}} \text {. }
$$


Hence, multiplying both sides of (28) by $v_{\vec{w}}(B)^{1 / \alpha-1 / q-1 / p}$, note that $1 / \alpha-1 / q-1 / p<0$ and $p / p_{i}=q / q_{i}=\alpha / \alpha_{i}$; by Lemma 8 and (29) we obtain

$$
\begin{aligned}
& v_{\vec{w}}(B)^{1 / \alpha-1 / q-1 / p}\left\|T(\vec{f}) \chi_{B}\right\|_{L_{v_{\vec{w}}}^{q}} \\
& \leq C \prod_{i=1}^{m} w_{i}(B)^{(1 / \alpha-1 / q-1 / p) q / q_{i} \|}\left\|T(\vec{f}) \chi_{B}\right\|_{L_{v_{\vec{w}}}^{q}} \\
& \leq C \prod_{i=1}^{m} w_{i}(B)^{1 / \alpha_{i}-1 / q_{i}-1 / p_{i}}\left\|T(\vec{f}) \chi_{B}\right\|_{L_{v_{\vec{w}}}^{q}} \\
& \leq C \prod_{i=1}^{m} w_{i}(2 B)^{1 / \alpha_{i}-1 / q_{i}-1 / p_{i}}\left\|f_{i} \chi_{2 B}\right\|_{L_{w_{i}}^{q_{i}}} \\
& \quad+C \sum_{j=1}^{m} \sum_{\sigma \in D_{j}^{m}}\left(\prod_{i \in \tau} w_{i}(2 B)^{1 / \alpha_{i}-1 / q_{i}-1 / p_{i}}\left\|f_{i} \chi_{2 B}\right\|_{L_{w_{i}}^{q_{i}}}\right) \\
& \quad \times \prod_{i \in \sigma}^{\infty} \frac{1+\gamma k}{2^{k n c_{q_{i}}}\left(1 / \alpha_{i}-1 / p_{i}+\beta\right)} w_{i}\left(2^{k+1} B\right)^{1 / \alpha_{i}-1 / q_{i}-1 / p_{i}} \\
& \quad \times\left\|f_{i} \chi_{2^{k+1} B}\right\|_{L_{w_{i}}^{q_{i}},}
\end{aligned}
$$

which combined with the fact that $\alpha_{i}<p_{i}$ for all $i \in\{1, \ldots, m\}$ leads to

$$
\|T(\vec{f})\|_{\left(L_{v_{\vec{w}}}^{q} L^{p}\right)^{\alpha}\left(\mathbb{R}^{n}\right)} \leq C \prod_{j=1}^{m}\left\|f_{j}\right\|_{\left(L_{w_{j}}, L^{q_{j}}\right)^{\alpha_{j}}\left(\mathbb{R}^{n}\right)^{\alpha_{j}}} .
$$

Theorem 3 is proved.

Proof of Theorem 4. For fixed $x \in B$, by linearity we can write

$$
\begin{aligned}
T_{\Sigma \vec{b}}(\vec{f})(x)= & T_{\Sigma \vec{b}}\left(\vec{f} \chi_{2 B}\right)(x) \\
& +\sum_{j=1}^{m} \sum_{\sigma \in D_{j}^{m}}\left(\sum_{\mu \in \sigma} T_{\vec{b}}^{\mu}\left(\overrightarrow{f^{\sigma}}\right)(x)\right. \\
& \left.+\sum_{\nu \in \tau} T_{\vec{b}}^{v}\left(\overrightarrow{f^{\sigma}}\right)(x)\right) .
\end{aligned}
$$

Invoking (18), (20)-(21) and Hölder's inequality, we have

$$
\begin{array}{r}
\left\|T_{\Sigma \vec{b}}(\vec{f}) \chi_{B}\right\|_{L_{v_{\vec{w}}}^{q}} \\
\leq C\left(\sum_{i=1}^{m}\left\|b_{i}\right\|_{\text {BMO }}\right) \prod_{i=1}^{m}\left\|f_{i} \chi_{2 B}\right\|_{L_{w_{i}}^{q_{i}}} \\
+C \sum_{j=1}^{m} \sum_{\sigma \in D_{j}^{m}} \sum_{\mu \in \sigma}\left(\prod_{i \in \tau}\left(\frac{w_{i}(B)}{w_{i}(2 B)}\right)^{1 / q_{i}}\right. \\
\left.\times\left\|f_{i} \chi_{2 B}\right\|_{L_{w_{i}}^{q_{i}}}\right)
\end{array}
$$

$$
\begin{aligned}
& \times\left(\prod_{i \in \sigma \backslash\{\mu\}} \sum_{k=1}^{\infty} \frac{1+\gamma k}{2^{k \beta}}\left(\frac{w_{i}(B)}{w_{i}\left(2^{k+1} B\right)}\right)^{1 / q_{i}}\right. \\
& \left.\times\left\|f_{i} \chi_{2^{k+1} B}\right\|_{L_{w_{i}}^{q_{i}}}\right) \\
& \times \sum_{k=1}^{\infty} \frac{1+\gamma k}{2^{k \beta}}\left\|b_{\mu}\right\|_{\text {BMO }}\left(\frac{w_{\mu}(B)}{w_{\mu}\left(2^{k+1} B\right)}\right)^{1 / q_{\mu}} \\
& \times\left\|f_{\mu} \chi_{2^{k+1} B}\right\|_{L_{w_{\mu}}^{q_{\mu}}} \\
& +C \sum_{j=1}^{m} \sum_{\sigma \in D_{j}^{m}} \sum_{v \in \tau}\left(\prod_{i \in \tau \mid\{v\}}\left(\frac{w_{i}(B)}{w_{i}(2 B)}\right)^{1 / q_{i}}\right. \\
& \left.\times\left\|f_{i} \chi_{2 B}\right\|_{L_{w_{i}}^{q_{i}}}\right) \\
& \times\left(\prod_{i \in \sigma} \sum_{k=1}^{\infty} \frac{1+\gamma k}{2^{k \beta}}\left(\frac{w_{i}(B)}{w_{i}\left(2^{k+1} B\right)}\right)^{1 / q_{i}}\right. \\
& \left.\times\left\|f_{i} \chi_{2^{k+1} B}\right\|_{L_{w_{i}}^{q_{i}}}\right) \\
& \times\left\|b_{\nu}\right\|_{\mathrm{BMO}}\left(\frac{w_{\nu}(B)}{w_{\nu}(2 B)}\right)^{1 / q_{v}}\left\|f_{\nu} \chi_{2 B}\right\|_{L_{w_{v}}^{q_{\nu}}} .
\end{aligned}
$$

By a similar argument as in getting (31), we can conclude that

$$
\begin{aligned}
& \left\|T_{\Sigma \vec{b}}(\vec{f})\right\|_{\left(L_{\vec{w},}^{q}, L^{p}\right)^{\alpha}\left(\mathbb{R}^{n}\right)} \\
& \quad \leq C\left(\sum_{j=1}^{m}\left\|b_{j}\right\|_{B M O\left(\mathbb{R}^{n}\right)}\right) \prod_{j=1}^{m}\left\|f_{j}\right\|_{\left(L_{w_{j}}^{q_{j}}, L^{p_{j}}\right)^{\alpha_{j}}\left(\mathbb{R}^{n}\right)^{n}},
\end{aligned}
$$

which completes the proof of Theorem 4.

Proof of Theorem 5. For fixed $x \in B$, we can write

$$
\begin{aligned}
T_{\Pi \vec{b}}(\vec{f})(x)= & T_{\Pi \vec{b}}\left(\vec{f} \chi_{2 B}\right)(x) \\
& +\sum_{j=1}^{m} \sum_{\sigma \in D_{j}^{m}} T_{\Pi \vec{b}}\left(\overrightarrow{f^{\sigma}}\right)(x) .
\end{aligned}
$$

Applying (22), (24) and Hölder's inequality, we get that

$$
\begin{aligned}
& \left\|T_{\Pi \vec{b}}(\vec{f}) \chi_{B}\right\|_{L_{v_{\vec{w}}}^{q}} \\
& \leq C\left(\prod_{i=1}^{m}\left\|b_{i}\right\|_{\mathrm{BMO}}\right) \prod_{i=1}^{m}\left\|f_{i} \chi_{2 \mathrm{~B}}\right\|_{L_{w_{i}}^{q_{i}}} \\
& \quad+C \sum_{j=1}^{m} \sum_{\sigma \in D_{j}^{m}} \sum_{\eta=0}^{m} \sum_{\sigma_{0} \in D_{\eta}^{m}}\left(\prod_{i \in \sigma_{0}}\left\|b_{i}\right\|_{\mathrm{BMO}}\right)\left(\prod_{i \in \tau}\left\|b_{i}\right\|_{\mathrm{BMO}}\right)
\end{aligned}
$$




$$
\begin{aligned}
& \times\left(\prod_{i \in \tau}\left(\frac{w_{i}(B)}{w_{i}(2 B)}\right)^{1 / q_{i}}\left\|f_{i} \chi_{2 B}\right\|_{L_{w_{i}}^{q_{i}}}\right) \\
& \times \prod_{i \in \sigma} \sum_{k=1}^{\infty} \frac{1+\gamma k}{2^{k \beta}}\left(\frac{w_{i}(B)}{w_{i}\left(2^{k+1} B\right)}\right)^{1 / q_{i}}\left\|f_{i} \chi_{2^{k+1} B}\right\|_{L_{w_{i}}^{q_{i}}}
\end{aligned}
$$

By similar arguments as in getting (31) again, we can deduce that

$$
\begin{aligned}
& \left\|T_{\Pi \vec{b}}(\vec{f})\right\|_{\left(L_{\vec{w}}^{q}, L^{p}\right)^{\alpha}\left(\mathbb{R}^{n}\right)} \\
& \quad \leq C\left(\prod_{j=1}^{m}\left\|b_{j}\right\|_{\mathrm{BMO}\left(\mathbb{R}^{n}\right)}\right) \prod_{j=1}^{m}\left\|f_{j}\right\|_{\left(L_{w_{j}}, L^{q_{j}}\right)^{\alpha_{j}}\left(\mathbb{R}^{n}\right)} .
\end{aligned}
$$

This completes the proof of Theorem 5 .

Proof of Theorem 6. For fixed $x \in B$, it is easy to check that

$$
\frac{1}{2}|z-y| \leq|z-x| \leq 2|z-y|, \quad \text { if } z \in(2 B)^{c} .
$$

Since $w_{i} \in A_{q_{i}}$, we have for any $i \in\{1, \ldots, m\}$ and $\beta \in \mathbb{N}$,

$$
\begin{gathered}
\int_{2 B}\left|f_{i}(z)\right| d z \leq C\left\|f_{i} \chi_{2 B}\right\|_{L_{w_{i}}^{q_{i}}}|2 B| w_{i}(2 B)^{-1 / q_{i}} \\
\int_{(2 B)^{c}} \frac{\left|f_{i}(z)\right|}{|y-z|^{n}} d z \\
\leq \sum_{k=1}^{\infty}\left|2^{k} B\right|^{-1} \int_{2^{k+1} B \backslash 2^{k} B}\left|f_{i}(z)\right| d z \\
\leq C \sum_{k=1}^{\infty}\left\|f_{i} \chi_{2^{k+1} B}\right\|_{L_{w_{i}}^{q_{i}}} w_{i}\left(2^{k+1} B\right)^{-1 / q_{i}} \\
\int_{(2 B)^{c}} \frac{\left|f_{i}(z)\right|}{|y-z|^{(\beta+1) n}} d z \\
\leq C \sum_{k=1}^{\infty}\left\|f_{i} \chi_{2^{k+1} B}\right\|_{L_{w_{i}}^{q_{i}}} \\
\times\left|2^{k+1} B\right|^{-\beta} w_{i}\left(2^{k+1} B\right)^{-1 / q_{i}}
\end{gathered}
$$

By Hölder's inequality, (25) and (38), writing $\sigma=\{\sigma(1)$, $\ldots, \sigma(j)\}$, we have

$$
\begin{aligned}
\left|T\left(\overrightarrow{f^{\sigma}}\right)(x)\right| \\
\leq C\left(\prod_{i \in \tau} \int_{2 B}\left|f_{i}(z)\right| d z\right) \\
\quad \times \int_{\left(\mathbb{R}^{n}\right)^{j}}\left(\sum_{i \in \sigma}\left|x-y_{i}\right|\right)^{-m n} \prod_{i \in \sigma}\left|f_{i}\left(y_{i}\right) \chi_{(2 B)^{c}}\left(y_{i}\right)\right| d y_{i}
\end{aligned}
$$

$$
\begin{aligned}
\leq & C\left(\prod_{i \in \tau} \int_{2 B}\left|f_{i}(z)\right| d z\right) \\
& \times\left(\prod_{i=1}^{j-1} \int_{(2 B)^{c}} \frac{\left|f_{\sigma(i)}(z)\right|}{|y-z|^{n}} d z\right) \int_{(2 B)^{c}} \frac{\left|f_{\sigma(j)}(z)\right|}{|y-z|^{(m-j+1) n}} d z .
\end{aligned}
$$

It follows from (39)-(42) that

$$
\begin{aligned}
\left|T\left(\frac{\overrightarrow{f^{\sigma}}}{\sigma}\right)(x)\right| & \\
\leq & C\left(\prod_{i \in \mathcal{\tau}}\left\|f_{i} \chi_{2 B}\right\|_{L_{w_{i}}^{q_{i}}}|2 B| w_{i}(2 B)^{-1 / q_{i}}\right) \\
& \times\left(\prod_{i=1}^{j-1} \sum_{k=1}^{\infty} w_{\sigma(i)}\left(2^{k+1} B\right)^{-1 / q_{\sigma(i)}}\left\|f_{\sigma(i)} \chi_{2^{k+1} B}\right\|_{L_{w \sigma(i)}^{q_{\sigma(i)}}}\right) \\
& \times \sum_{k=1}^{\infty}\left|2^{k+1} B\right|^{-m+j} w_{\sigma(j)}\left(2^{k+1} B\right)^{-1 / q_{\sigma(j)}} \\
\leq & C\left(\prod_{i \in \tau}\left\|f_{i} \chi_{2 B}\right\|_{L_{w_{i}}^{q_{i}}} w_{i}(2 B)^{-1 / q_{i}}\right) \\
& \times\left(\prod_{i=1}^{j-1} \sum_{k=1}^{\infty} w_{\sigma(i)}\left(2^{k+1} B\right)^{-1 / q_{\sigma(i)}}\left\|f_{\sigma(i)} \chi_{2^{k+1} B}\right\|_{L_{w_{\sigma(i)}}^{q_{\sigma(i)}}}\|\|_{L_{w_{\sigma(j)}}^{q_{\sigma(j)}}}\right) \\
& \times \sum_{k=1}^{\infty} 2^{-k(m-j)} w_{\sigma(j)}\left(2^{k+1} B\right)^{-1 / q_{\sigma(j)}} \\
& \times f_{\sigma(j)} \chi_{2^{k+1} B} \|_{L_{w_{\sigma(j)}}^{q_{\sigma(j)}}} \\
& (2)
\end{aligned}
$$

This implies (17) in the case of that $\beta=0$ and $\gamma=0$ or $\beta=$ $m-j$ and $\gamma=0$.

For $T_{\Sigma \vec{b}}, \sigma \in D_{j}^{m}$ and $i \in \sigma$, we have from (25) and (38) that

$$
\begin{aligned}
& \left|T_{\vec{b}}^{i}\left(\overrightarrow{\overrightarrow{f^{\sigma}}}\right)(x)\right| \\
& \leq C \int_{\left(\mathbb{R}^{n}\right)^{m}}\left|b_{i}(x)-b_{i}\left(y_{i}\right)\right| \\
& \quad \times \prod_{k=1}^{m}\left|\tilde{f}_{k}\left(y_{k}\right)\right|\left(\sum_{k=1}^{m}\left|y-y_{k}\right|\right)^{-m n} d y_{1} \cdots d y_{m} \\
& \leq C\left(\prod_{k \in \tau} \int_{2 B}\left|f_{k}(z)\right| d z\right) \int_{(2 B)^{c}} \frac{\left|\left(b_{i}(x)-b_{i}(z)\right) f_{i}(z)\right|}{|x-z|^{n}} d z \\
& \quad \times \int_{\left(\mathbb{R}^{n}\right)^{j-1}}\left(\sum_{k \in \sigma \backslash\{i\}}\left|y-y_{k}\right|\right)^{-(m-1) n} \\
& \quad \times \prod_{k \in \sigma \backslash\{i\}}\left|f_{k}\left(y_{k}\right) \chi_{(2 B)^{c}}\left(y_{k}\right)\right| d y_{k} .
\end{aligned}
$$


Since $w_{v} \in A_{q_{v}}$, thus $w^{1-q_{v}^{\prime}} \in A_{q_{v}^{\prime}}$ with $q_{v}^{\prime}=q_{v} /\left(q_{v}-1\right)$. By the properties of functions in $\mathrm{BMO}\left(\mathbb{R}^{n}\right)$ and Hölder's inequality, we have for any ball $Q$ and $v \in\{1, \ldots, m\}$,

$$
\begin{aligned}
\int_{\mathrm{Q}} & \left|b_{v}(z)-\left(b_{v}\right)_{\mathrm{Q}}\right|\left|f_{v}(z)\right| d z \\
\leq & \left(\int_{\mathrm{Q}}\left|f_{v}(z)\right|^{q_{v}} w(z) d z\right)^{1 / q} \\
& \times\left(\int_{\mathrm{Q}}\left|b_{v}(z)-\left(b_{v}\right)_{\mathrm{Q}}\right|^{q_{v}^{\prime}} w_{v}^{1-q_{v}^{\prime}}(z) d z\right)^{1 / q_{v}^{\prime}} \\
\leq & C\left\|f_{v} \chi_{\mathrm{Q}}\right\|_{L_{w_{v}}}\left\|b_{v}\right\|_{\mathrm{BMO}}|\mathrm{Q}| w_{v}(\mathrm{Q})^{-1 / q_{v}} .
\end{aligned}
$$

It follows from (39) and (45) that for any $v \in\{1, \ldots, m\}$ and $1<q_{v}<\infty$,

$$
\begin{gathered}
\int_{(2 B)^{c}} \frac{\left|\left(b_{\nu}(x)-b_{\nu}(z)\right) f_{\nu}(z)\right|}{|x-z|^{n}} d z \\
\leq C \sum_{k=1}^{\infty} \int_{2^{k+1} B \mid 2^{k} B} \frac{\left|\left(b_{\nu}(x)-b_{\nu}(z)\right) f_{\nu}(z)\right|}{|y-z|^{n}} d z \\
\leq C \sum_{k=1}^{\infty}\left(\left|b_{\nu}(x)-\left(b_{\nu}\right)_{2^{k+1} B}\right|+k\left\|b_{\nu}\right\|_{\mathrm{BMO}}\right) \\
\times w_{\nu}\left(2^{k+1} B\right)^{-1 / q_{\nu}}\left\|f_{\nu} \chi_{2^{k+1} B}\right\|_{L_{w_{\nu}}^{q_{\nu}}} .
\end{gathered}
$$

Let $\mu=\sigma\left(i_{0}\right)$ for some $i_{0} \in\{1, \ldots, j\}$. We now consider two cases:

Case $1\left(i_{0} \neq j\right)$. We have

$$
\begin{aligned}
\left.\mid T_{\vec{b}}^{\mu}(\overrightarrow{\vec{f}})^{\sigma}\right)(x) \mid & \\
\leq & C\left(\prod_{i \in \tau}\left\|f_{i} \chi_{2 B}\right\|_{L_{w_{i}}^{q_{i}}}|2 B| w_{i}(2 B)^{-1 / q_{i}}\right) \\
& \times\left(\prod_{i \in\{1, \ldots, j-1\} \backslash\left\{i_{0}\right\}} \int_{(2 B)^{c}} \frac{\left|f_{\sigma(i)}(z)\right|}{|y-z|^{n}} d z\right) \\
& \times \int_{(2 B)^{c}} \frac{\left|f_{\sigma(j)}(z)\right|}{|y-z|^{(m-j+1) n}} d z \\
& \times \sum_{k=1}^{\infty}\left(\left|b_{\mu}(x)-\left(b_{\mu}\right)_{2^{k+1} B}\right|+k\left\|b_{\mu}\right\|_{\mathrm{BMO}}\right) \\
\leq & C\left(\prod_{i \in \tau}\left\|f_{i} \chi_{2 B}\right\|_{L_{w_{i}}^{q_{i}}} w(2 B)^{-1 / q_{i}}\right)
\end{aligned}
$$

$$
\begin{aligned}
& \times\left(\prod_{i \in\{1, \ldots, j-1\}\left\{i_{0}\right\}} \sum_{k=1}^{\infty} w_{\sigma(i)}\left(2^{k+1} B\right)^{-1 / q_{\sigma(i)}}\left\|f_{\sigma(i)} \chi_{2^{k+1} B}\right\|_{L_{w_{\sigma}(i)}^{q_{\sigma(i)}}}\right) \\
& \times \sum_{k=1}^{\infty} 2^{-k(m-j)} w_{\sigma(j)}\left(2^{k+1} B\right)^{-1 / q_{\sigma(j)}} \\
& \quad \times\left\|f_{\sigma(j)} \chi_{2^{k+1} B}\right\|_{L_{w_{\sigma(j)}}^{q_{\sigma(j)}}} \\
& \times \sum_{k=1}^{\infty}\left(\left|b_{\mu}(x)-\left(b_{\mu}\right)_{2^{k+1} B}\right|+k\left\|b_{\mu}\right\|_{\mathrm{BMO}}\right) \\
& \quad \times w_{\mu}\left(2^{k+1} B\right)^{-1 / q_{\mu}}\left\|f_{\mu} \chi_{2^{k+1} B}\right\|_{L_{w_{\mu}}^{q_{\mu}}},
\end{aligned}
$$

which satisfies (20) in the case of that $\beta=0$ and $\gamma=0$ or $\beta=m-j$ and $\gamma=0$.

Case $2\left(i_{0}=j\right)$. We have

$$
\begin{aligned}
\left|T_{\vec{b}}^{\mu}\left(\overrightarrow{f^{\sigma}}\right)(x)\right| & \\
\leq & C\left(\prod_{i \in \tau}\left\|f_{i} \chi_{2 B}\right\|_{L_{w_{i}}^{q_{i}}}|2 B| w_{i}(2 B)^{-1 / q_{i}}\right) \\
& \times\left(\prod_{i \in\{1, \ldots, j-2\}} \int_{(2 B)^{c}} \frac{\left|f_{\sigma(i)}(z)\right|}{|y-z|^{n}} d z\right) \\
& \times \int_{(2 B)^{c}} \frac{\left|f_{\sigma(j-1)}(z)\right|}{|y-z|^{(m-j+1) n}} d z \\
& \times \sum_{k=1}^{\infty}\left(\left|b_{\mu}(x)-\left(b_{\mu}\right)_{2^{k+1} B}\right|+k\left\|b_{\mu}\right\|_{\text {BMO }}\right) \\
\leq & C\left(\prod_{i \in \tau}\left\|f_{i} \chi_{2 B}\right\|_{L_{w_{i}}^{q_{i}}} w_{i}(2 B)^{-1 / q_{i}}\right) \\
& \times\left(w_{\mu}\left(2^{k+1} B\right)^{-1 / q_{\mu}}\left\|f_{\mu} \chi_{2^{k+1} B}\right\|_{L_{w_{\mu}}^{q_{\mu}}}\right. \\
& \times \sum_{i \in\{1, \ldots, j-2\}} \sum_{k=1}^{\infty}\left(\left|b_{\mu}(x)-\left(b_{\mu}\right)_{2^{k+1} B}\right|+k\left\|b_{\mu}\right\|_{B M O}\right) \\
& \left.\times \sum_{k=1}^{\infty} 2^{-k(m-j)} w_{\sigma(j-1)}\left(2^{k+1} B\right)^{-1 / q \sigma(i)}\left\|f_{\sigma(i)} \chi_{2^{k+1} B}\right\|_{L_{w_{\sigma(i)}} q_{(i)}} B\right)^{-1 / q_{\sigma(j-1)}}
\end{aligned}
$$


which satisfies (20) in the case of that $\beta=0$ and $\gamma=0$ or $\beta=m-j$.

For $v \in \tau$, it follows from (25) and (38) that

$$
\begin{aligned}
& \left|T_{\vec{b}}^{v}\left(\overrightarrow{f^{\sigma}}\right)(x)\right| \\
& \leq C \int_{\left(\mathbb{R}^{n}\right)^{m}}\left|b_{\nu}(x)-b_{\nu}\left(y_{\nu}\right)\right| \\
& \quad \times\left(\sum_{k=1}^{m}\left|y-y_{k}\right|\right)^{-m n} \prod_{k=1}^{m}\left|\tilde{f}_{k}\left(y_{k}\right)\right| d y_{1} \cdots d y_{m} \\
& \leq C\left(\prod_{i \in \tau \mid\{v\}} \int_{2 B}\left|f_{i}(z)\right| d z\right) \prod_{i=1}^{j-1} \int_{(2 B)^{c}} \frac{\left|f_{\sigma(i)}(z)\right|}{|y-z|^{n}} d z \\
& \quad \times \int_{(2 B)^{c}} \frac{\left|f_{\sigma(j)}(z)\right|}{|y-z|^{(m-j+1) n}} d z \\
& \quad \times \int_{2 B}\left|\left(b_{\nu}(x)-b_{\nu}(z)\right) f_{\nu}(z)\right| d z .
\end{aligned}
$$

This together with (39)-(41), (45) and Hölder's inequality leads to

$$
\begin{aligned}
& \left|T_{\vec{b}}^{v}\left(\overrightarrow{f^{\sigma}}\right)(x)\right| \\
& \leq C\left(\prod_{i \in \mathcal{T} \backslash\{\nu\}}\left\|f_{i} \chi_{2 B}\right\|_{L_{w_{i}}^{q_{i}}}|2 B| w_{i}(2 B)^{-1 / q_{i}}\right) \\
& \times \prod_{i=1}^{j-1} \sum_{k=1}^{\infty} w_{\sigma(i)}\left(2^{k+1} B\right)^{-1 / q_{\sigma(i)}}\left\|f_{\sigma(i)} \chi_{2^{k+1} B}\right\|_{L_{w_{\sigma(i)}}^{q_{\sigma(i)}}} \\
& \times \sum_{k=1}^{\infty}\left|2^{k+1} B\right|^{-m+j} w_{\sigma(j)}\left(2^{k+1} B\right)^{-1 / q_{\sigma(j)}}\left\|f_{\sigma(j)} \chi_{2^{k+1} B}\right\|_{L_{w_{\sigma(j)}}^{q_{\sigma(j)}}} \\
& \times\left(\left|b_{\nu}(x)-\left(b_{\nu}\right)_{2 B}\right|+\left\|b_{\nu}\right\|_{\mathrm{BMO}}\right) \\
& \times|2 B| w_{\nu}(2 B)^{-1 / q_{\nu}}\left\|f_{\nu} \chi_{2 B}\right\|_{L_{w_{\nu}}^{q_{\nu}}} \\
& \leq C\left(\prod_{i \in \mathcal{T} \backslash\{v\}}\left\|f_{i} \chi_{2 B}\right\|_{L_{w_{i}}^{q_{i}}} w_{i}(2 B)^{-1 / q_{i}}\right) \\
& \times \prod_{i=1}^{j-1} \sum_{k=1}^{\infty} w_{\sigma(i)}\left(2^{k+1} B\right)^{-1 / q_{\sigma(i)}}\left\|f_{\sigma(i)} \chi_{2^{k+1} B}\right\|_{L_{w_{\sigma(i)}}^{q_{\sigma(i)}}} \\
& \times \sum_{k=1}^{\infty} 2^{-k(m-j)} w_{\sigma(j)}\left(2^{k+1} B\right)^{-1 / q_{\sigma(j)}}\left\|f_{\sigma(j)} \chi_{2^{k+1} B}\right\|_{L_{w_{\sigma(j)}}^{q_{\sigma(j)}}} \\
& \times\left(\left|b_{\nu}(x)-\left(b_{\nu}\right)_{2 B}\right|+\left\|b_{\nu}\right\|_{\text {BMO }}\right) \\
& \times w_{\nu}(2 B)^{-1 / q_{\nu}}\left\|f_{\nu} \chi_{2 B}\right\|_{L_{w_{\nu}}^{q_{\nu}}},
\end{aligned}
$$

which satisfies (21) in the case of that $\beta=0$ and $\gamma=0$ or $\beta=m-j$ and $\gamma=0$.
For $T_{\Pi \vec{b}}, j \in\{1, \ldots, m\}, \sigma \in D_{j}^{m}, \sigma=\{\sigma(1), \ldots, \sigma(j)\}$ and $\tau=\{1, \ldots, m\} \backslash \sigma$, we have

$$
\begin{aligned}
& T_{\Pi \vec{b}}(\overrightarrow{\vec{f}})(x) \\
& =\int_{\left(\mathbb{R}^{n}\right)^{m}} \prod_{i=1}^{m}\left(b_{i}(x)-b_{i}(y)\right) K\left(x, y_{1}, \ldots, y_{m}\right) \\
& \quad \times \prod_{i \in \sigma} f_{i}\left(y_{i}\right) \chi_{(2 B)^{c}}\left(y_{i}\right) \prod_{i \in \tau} f_{i}\left(y_{i}\right) \chi_{2 B}\left(y_{i}\right) d y_{1} \cdots d y_{m} \\
& \quad:=\sum_{\eta=0}^{m} \sum_{\sigma_{0} \in D_{\eta}^{m}} T_{j, \sigma_{0}}(\vec{f})(x),
\end{aligned}
$$

where

$$
\begin{aligned}
T_{j, \sigma_{0}}(\vec{f})(x) \\
:=\prod_{i \in \sigma_{0}}\left(b_{i}(x)-\left(b_{i}\right)_{2 B}\right) \\
\quad \times \int_{\left(\mathbb{R}^{n}\right)^{m}} \prod_{i \in \tau_{0}}\left(\left(b_{i}\right)_{2 B}-b_{i}\left(y_{i}\right)\right) K\left(x, y_{1}, \ldots, y_{m}\right) \\
\quad \times \prod_{i \in \sigma} f_{i}\left(y_{i}\right) \chi_{(2 B)^{c}}\left(y_{i}\right) \prod_{i \in \tau} f_{i}\left(y_{i}\right) \chi_{2 B}\left(y_{i}\right) d y_{1} \cdots d y_{m} .
\end{aligned}
$$

For fixed $x \in B, \eta \in\{0, \ldots, m\}$ and $\sigma_{0} \in D_{\eta}^{m}$, we set

$$
\tau_{1}:=\tau \cap \tau_{0}, \quad \tau_{2}:=\tau_{0} \backslash \tau, \quad \tau_{3}:=\tau \backslash \tau_{0} .
$$

Then by (25) and (38), we have

$$
\begin{aligned}
& \left|T_{j, \sigma_{0}}(\vec{f})(x)\right| \\
& \leq C\left(\prod_{i \in \sigma_{0}}\left|b_{i}(x)-\left(b_{i}\right)_{2 B}\right|\right) \\
& \quad \times \int_{\left(\mathbb{R}^{n}\right)^{m}}\left(\prod_{i \in \tau_{1}}\left|\left(b_{i}\left(y_{i}\right)-\left(b_{i}\right)_{2 B}\right) f_{i}\left(y_{i}\right) \chi_{2 B}\left(y_{i}\right)\right|\right) \\
& \quad \times\left(\prod_{i \in \tau_{2}}\left|b_{i}\left(y_{i}\right)-\left(b_{i}\right)_{2 B}\right|\right)\left(\prod_{i \in \tau_{3}}\left|f_{i}\left(y_{i}\right) \chi_{2 B}\left(y_{i}\right)\right|\right) \\
& \quad \times \prod_{i \in \sigma}\left|f_{i}\left(y_{i}\right) \chi_{(2 B)^{c}}\left(y_{i}\right)\right|\left|K\left(x, y_{1}, \ldots, y_{m}\right)\right| d y_{1} \cdots d y_{m} \\
& \leq C \\
& \quad \times\left(\prod_{i \in \sigma_{0}}\left|b_{i}(x)-\left(b_{i}\right)_{2 B}\right|\right) \\
& \quad \times\left(\prod_{i \in \tau_{1}} \int_{2 B}\left|b_{i}(z)-\left(b_{i}\right)_{2 B}\right|\left|f_{i}(z)\right| d z\right)
\end{aligned}
$$




$$
\begin{aligned}
& \times\left(\prod_{i \in \tau_{3}} \int_{2 B}\left|f_{i}(z)\right| d z\right) \\
& \times\left(\prod_{i \in \tau_{2}} \int_{(2 B)^{c}} \frac{\left|b_{i}(z)-\left(b_{i}\right)_{2 B}\right|\left|f_{i}(z)\right|}{|y-z|^{n}} d z\right) \\
& \times \prod_{i \in \sigma \backslash\left(\tau_{2} \cup\{\sigma(j)\}\right)} \int_{(2 B)^{c}} \frac{\left|f_{i}(z)\right|}{|y-z|^{n}} d z \\
& \times \int_{(2 B)^{c}} \frac{\left|f_{\sigma(j)}(z)\right|}{|y-z|^{(m-j+1) n}} d z .
\end{aligned}
$$

It is easy to check that

$$
\begin{gathered}
\int_{(2 B)^{c}} \frac{\left|\left(b_{i}(z)-\left(b_{i}\right)_{2 B}\right) f_{i}(z)\right|}{|y-z|^{n}} d z \\
\leq C \sum_{k=1}^{\infty}(k+1)\left\|b_{i}\right\|_{\mathrm{BMO}} w_{i}\left(2^{k+1} B\right)^{-1 / q_{i}} \\
\times\left\|f_{i} \chi_{2^{k+1} B}\right\|_{L_{w_{i}}^{q_{i}}}
\end{gathered}
$$

for any $i \in\{1, \ldots, m\}$. This combining (39)-(41), (45) with (54) yields that

$$
\begin{aligned}
\left|T_{j, \sigma_{0}}(\vec{f})(x)\right| & \\
\leq & C\left(\prod_{i \in \sigma_{0}}\left|b_{i}(x)-\left(b_{i}\right)_{2 B}\right|\right)\left(\prod_{i \in \tau_{0}}\left\|b_{i}\right\|_{\text {BMO }}\right) \\
& \times\left(\prod_{i \in \tau_{1}} w_{i}(2 B)^{-1 / q_{i}}\left\|f_{i} \chi_{2 B}\right\|_{L_{w_{i}}^{q_{i}}}\right) \\
& \times\left(\prod_{i \in \tau_{3}} w_{i}(2 B)^{-1 / q_{i}}\left\|f_{i} \chi_{2 B}\right\|_{L_{w_{i}}^{q_{i}}}\right) \\
& \times\left(\prod_{i \in \tau_{2}} \sum_{k=1}^{\infty}(k+1) w_{i}\left(2^{k+1} B\right)^{-1 / q_{i}}\left\|f_{i} \chi_{2^{k+1} B}\right\|_{L_{w_{i}}^{q_{i}}}\right) \\
& \times \prod_{i \in \sigma \backslash\left(\tau_{2} \cup\{\sigma(j)\}\right) k=1}^{\infty} \sum_{k\left(2^{k+1} B\right)^{-1 / q_{i}}\left\|f_{i} \chi_{2^{k+1} B}\right\|_{L_{w}^{q_{i}}}}^{\infty} \sum_{k=1}^{-k(m-j)} w\left(2^{k+1} B\right)^{-1 / q_{\sigma(j)}}\left\|f_{\sigma(j)} \chi_{2^{k+1} B}\right\|_{L_{w_{\sigma(j)} q_{(j)}}}
\end{aligned}
$$

This together with (51) implies (24) in the case of that $\beta=0$ and $\gamma=1$, or $\beta=0$ and $\gamma=0$, or $\beta=m-j$ and $\gamma=0$, and completes the proof of Theorem 6 .

\section{Applications}

3.1. On the Multilinear Calderón-Zygmund Operators. An mlinear operator $T$ associated with $K$ is said to be an $m$-linear Calderón-Zygmund operator if, for some $1 \leq q_{j}<\infty$, it extends to a bounded multilinear operator from $L^{q_{1}} \times \cdots \times L^{q_{m}}$ to $L^{q}$, where $1 / q=1 / q_{1}+\cdots+1 / q_{m}$ and the kernel $K$ satisfies (25) and the regularity conditions

$$
\begin{aligned}
& \left|K\left(y_{0}, \ldots, y_{j}, \ldots, y_{m}\right)-K\left(y_{0}, \ldots, y_{j}^{\prime}, \ldots, y_{m}\right)\right| \\
& \quad \leq \frac{A\left|y_{j}-y_{j}^{\prime}\right|^{\epsilon}}{\left(\sum_{k, l=0}^{m}\left|y_{k}-y_{l}\right|\right)^{m n+\epsilon}}
\end{aligned}
$$

for some $\epsilon>0$ and all $0 \leq j \leq m$, whenever $\left|y_{j}-y_{j}^{\prime}\right| \leq$ $1 / 2 \max _{0 \leq k \leq m}\left|y_{j}-y_{k}\right|$. We denote by $m-\operatorname{CZK}(A, \epsilon)$ the collection of all kernels $K$ satisfying (25) and (57).

As is well known, the multilinear version of the CalderónZygmund theory originated in the works of Coifman and Meyer in the 1970s; see, for example, [13, 14], and it was oriented towards the study of the Calderón commutator. Later on the topic was retaken by several authors, including Christ and Journé [15], Kenig and Stein [16], and Grafakos and Torres [17, 18]. Moreover, commutators of multilinear singular integral operators with $\mathrm{BMO}$ functions have been the subject of many recent articles (see [11, 19-21] et al.). The following results, which will be used in the next theorem, follow from $[11,20]$.

Lemma 9 (cf. $[11,20])$. Let $m \in \mathbb{N}$ with $m \geq 1$ and $T$ be an $m$-linear Calderón-Zygmund operator. Let $1 / p=\sum_{i=1}^{m} 1 / p_{i}$ with $1<p, p_{j}<\infty$, and $j=1, \ldots, m$. Suppose that $\vec{b}=\left(b_{1}, \ldots, b_{m}\right) \in B M O^{m}$ and $\vec{w}=\left(w_{1}, \ldots, w_{m}\right)$ satisfies the $A_{\vec{p}}$ condition. Then

$$
\begin{aligned}
& \|T(\vec{f})\|_{L_{v_{\vec{w}}}^{p_{\vec{w}}}\left(\mathbb{R}^{n}\right)} \leq C \prod_{j=1}^{m}\left\|f_{j}\right\|_{L_{w_{j}}^{p_{j}}\left(\mathbb{R}^{n}\right)}, \\
& \left\|T_{\Sigma \vec{b}}(\vec{f})\right\|_{L_{v_{\vec{w}}}^{p^{\prime}}\left(\mathbb{R}^{n}\right)} \leq C\left(\sum_{j=1}^{m}\left\|b_{j}\right\|_{B M O\left(\mathbb{R}^{n}\right)}\right) \prod_{j=1}^{m}\left\|f_{j}\right\|_{L_{w_{j}}^{p_{j}}\left(\mathbb{R}^{n}\right)}, \\
& \left\|T_{\Pi \vec{b}}(\vec{f})\right\|_{L_{v_{\vec{w}}}^{p}\left(\mathbb{R}^{n}\right)} \leq C\left(\prod_{j=1}^{m}\left\|b_{j}\right\|_{B M O\left(\mathbb{R}^{n}\right)}\right) \prod_{j=1}^{m}\left\|f_{j}\right\|_{L_{w_{j}}^{p_{j}}\left(\mathbb{R}^{n}\right)^{n}},
\end{aligned}
$$

where $v_{\vec{w}}=\prod_{j=1}^{m} w_{j}^{p / p_{j}}$.

This lemma together with Theorems 3-6 directly leads to the following result.

Theorem 10. Let $m \in \mathbb{N}$ with $m \geq 2$ and $T$ be an $m$-linear Calderón-Zygmund operator. Let $1<q_{j} \leq \alpha_{j}<p_{j}<$ $\infty(j=1, \ldots, m)$ satisfy $1 / q=\sum_{i=1}^{m} 1 / q_{i}, 1 / \alpha=\sum_{i=1}^{m} 1 / \alpha_{i}$, $1 / p=\sum_{i=1}^{m} 1 / p_{i}$, and $p / p_{j}=q / q_{j}=\alpha / \alpha_{j}(j=1, \ldots, m)$. 
Suppose that $\vec{w}=\left(w_{1}, \ldots, w_{m}\right) \in \prod_{j=1}^{m} A_{q_{j}}, v_{\vec{w}}=\prod_{j=1}^{m} w_{j}^{q / q_{j}}$, and $\vec{b}=\left(b_{1}, \ldots, b_{m}\right) \in B M O^{m}$. Then

$$
\begin{aligned}
& \|T(\vec{f})\|_{\left(L_{v_{\vec{w}}}^{q}, L^{p}\right)^{\alpha}\left(\mathbb{R}^{n}\right)} \leq C \prod_{j=1}^{m}\left\|f_{j}\right\|_{\left(L_{w_{j}}^{q_{j}}, L^{p_{j}}\right)^{\alpha_{j}}\left(\mathbb{R}^{n}\right)^{n}}, \\
& \left\|T_{\Sigma \vec{b}}(\vec{f})\right\|_{\left(L_{v_{\vec{w}}}^{q}, L^{p}\right)^{\alpha}\left(\mathbb{R}^{n}\right)} \\
& \leq C\left(\sum_{j=1}^{m}\left\|b_{j}\right\|_{B M O\left(\mathbb{R}^{n}\right)}\right) \prod_{j=1}^{m}\left\|f_{j}\right\|_{\left(L_{w_{j}}^{\left.q_{j}, L^{p_{j}}\right)^{\alpha_{j}}}\left(\mathbb{R}^{n}\right)^{\prime}\right.} \\
& \left\|T_{\Pi \vec{b}}(\vec{f})\right\|_{\left(L_{v_{\vec{w}}}^{q}, L^{p}\right)^{\alpha}\left(\mathbb{R}^{n}\right)} \\
& \leq C\left(\prod_{j=1}^{m}\left\|b_{j}\right\|_{B M O\left(\mathbb{R}^{n}\right)}\right) \prod_{j=1}^{m}\left\|f_{j}\right\|_{\left(L_{w_{j}}^{q_{j}}, L^{p_{j}}\right)^{\alpha_{j}}\left(\mathbb{R}^{n}\right)^{\prime}},
\end{aligned}
$$

where $C$ depends only on $m, n, p, q, \alpha$ and $[w]_{A_{q_{j}}}$.

Furthermore, by Remark 7 and Lemma 9, we have the following.

Theorem 11. Let $m \in \mathbb{N}$ with $m \geq 2$ and $T$ be an $m$-linear Calderón-Zygmund operator. Let $1<q_{j} \leq \alpha_{j}<p_{j}<\infty(j=$ $1, \ldots, m)$ satisfy $1 / q=\sum_{i=1}^{m} 1 / q_{i}, 1 / \alpha=\sum_{i=1}^{m} 1 / \alpha_{i}$ and $1 / p=$ $\sum_{i=1}^{m} 1 / p_{i}$. Assume that $w \in A_{q}$ and $\vec{b}=\left(b_{1}, \ldots, b_{m}\right) \in B M O^{m}$. Then

$$
\begin{aligned}
& \|T(\vec{f})\|_{\left(L_{w}^{q}, L^{p}\right)^{\alpha}\left(\mathbb{R}^{n}\right)} \leq C \prod_{j=1}^{m}\left\|f_{j}\right\|_{\left(L_{w}^{q_{j}}, L^{p_{j}}\right)^{\alpha_{j}}\left(\mathbb{R}^{n}\right)}, \\
& \left\|T_{\Sigma \vec{b}}(\vec{f})\right\|_{\left(L_{w}^{q}, L^{p}\right)^{\alpha}\left(\mathbb{R}^{n}\right)} \\
& \quad \leq C\left(\sum_{j=1}^{m}\left\|b_{j}\right\|_{B M O\left(\mathbb{R}^{n}\right)}\right) \prod_{j=1}^{m}\left\|f_{j}\right\|_{\left(L_{w}^{q_{j}}, L^{p_{j}}\right)^{\alpha_{j}}\left(\mathbb{R}^{n}\right)^{n}}, \\
& \left.\left\|T_{\Pi \vec{b}}(\vec{f})\right\|_{\left(L_{w}^{q}, L^{p}\right)^{\alpha}\left(\mathbb{R}^{n}\right)}\right) \prod_{j=1}^{m}\left\|f_{j}\right\|_{\left(L_{w}^{q_{j}}, L^{p_{j}}\right)^{\alpha_{j}}\left(\mathbb{R}^{n}\right)^{n}},
\end{aligned}
$$

where $C$ depends only on $m, n, p, q, \alpha$ and $[w]_{A_{q}}$.

3.2. On the Multilinear Singular Integrals with Nonsmoothness Kernels. Let $\left\{A_{t}\right\}_{t>0}$ be a class of integral operators, which play the roles of approximate identities (see [22]). We always assumed that the operators $A_{t}$ are associated with kernels $a_{t}(x, y)$ in the sense that for all $f \in \bigcup_{1 \leq p \leq \infty} L^{p}$ and $x \in \mathbb{R}^{n}$,

$$
A_{t} f(x)=\int_{\mathbb{R}^{n}} a_{t}(x, y) f(y) d y,
$$

and the kernels $a_{t}(x, y)$ satisfy the following conditions:

$$
\left|a_{t}(x, y)\right| \leq h_{t}(x, y):=t^{-n / s} h\left(\frac{|x-y|}{t^{1 / s}}\right),
$$

where $s$ is a positive fixed constant and $h$ is a positive, bounded, decreasing function satisfying that for some $\eta>0$,

$$
\lim _{r \rightarrow \infty} r^{n+\eta} h\left(r^{s}\right)=0 .
$$

Recall that the $j$ th transpose $T^{*, j}$ of the $m$ th linear operator $T$ is defined via

$$
\begin{aligned}
& \left\langle T^{*, j}\left(f_{1}, \ldots, f_{m}\right), g\right\rangle \\
& \quad=\left\langle T\left(f_{1}, \ldots, f_{j-1}, h, f_{j+1}, \ldots, f_{m}\right), f_{j}\right\rangle
\end{aligned}
$$

for all $f_{1}, \ldots, f_{m}, h$ in $\delta\left(\mathbb{R}^{n}\right)$. Notice that the kernel $K^{*, j}$ of $T^{*, j}$ is related to the kernel $K$ of $T$ via the identity

$$
\begin{aligned}
& K^{*, j}\left(x, y_{1}, \ldots, y_{j-1}, y_{j}, y_{j+1}, \ldots, y_{m}\right) \\
& \quad=K\left(y_{j}, y_{1}, \ldots, y_{j-1}, x, y_{j+1}, \ldots, y_{m}\right) .
\end{aligned}
$$

If an $m$-linear operator $T$ maps a product of Banach spaces $X_{1} \times \cdots \times X_{m}$ to another Banach space $X$, then the transpose $T^{*, j}$ maps $X_{1} \times \cdots \times X_{j-1} \times X \times X_{j+1} \times \cdots \times X_{m}$ to $X_{j}$. Moreover, the norm of $T$ and $T^{*, j}$ is equal. To maintain uniform notation, we may occasionally denote $T$ by $T^{*, 0}$ and $K$ by $K^{*, 0}$.

Assumption 12. Assume that for each $i \in\{1, \ldots, m\}$, there exist operators $\left\{A_{t}^{(i)}\right\}_{t>0}$ with kernels $a_{t}^{(i)}(x, y)$ that satisfy conditions (62)-(63) with constants $s, \eta$ and that for every $j \in\{0,1, \ldots, m\}$, there exist kernels $K_{t}^{*, j,(i)}\left(x, y_{1}, \ldots, y_{m}\right)$ such that

$$
\begin{aligned}
& \left\langle T^{*, j}\left(f_{1}, \ldots, A_{t}^{(i)} f_{i}, \ldots, f_{m}\right), f\right\rangle \\
& =\int_{\mathbb{R}^{n}} \int_{\left(\mathbb{R}^{n}\right)^{m}} K_{t}^{*, j,(i)}\left(x, y_{1}, \ldots, y_{m}\right) \\
& \quad \times f_{1}\left(y_{1}\right) \cdots f_{m}\left(y_{m}\right) g(x) d y_{1} \cdots d y_{m} d x
\end{aligned}
$$

for all $f_{1}, \ldots, f_{m}$ in $\mathcal{S}\left(\mathbb{R}^{n}\right)$ with $\bigcap_{k=1}^{m} \operatorname{supp}\left(f_{k}\right) \cap \operatorname{supp}(g)=$ $\emptyset$. Also assume that there exists a function $\phi \in \mathscr{C}(\mathbb{R})$ with $\operatorname{supp} \phi \subset[-1,1]$ and a constant $\epsilon>0$ so that for every $j \epsilon$ $\{0, \ldots, m\}$ and every $i \in\{1, \ldots, m\}$, we have

$$
\begin{aligned}
\mid K^{*, j} & \left(x, y_{1}, \ldots, y_{m}\right)-K^{*, j,(i)}\left(x, y_{1}, \ldots, y_{m}\right) \mid \\
\leq & \frac{A}{\left(\sum_{k=1}^{m}\left|x-y_{k}\right|\right)^{m n}} \\
& \times \sum_{k=1, k \neq i}^{m} \phi\left(\frac{\left|y_{i}-y_{k}\right|}{t^{1 / s}}\right)+\frac{A t^{\epsilon / s}}{\left(\sum_{k=1}^{m}\left|x-y_{k}\right|\right)^{m n+\epsilon}},
\end{aligned}
$$

whenever $t^{1 / s} \leq\left|x-y_{i}\right| / 2$.

We say that $T$ is an $m$-linear operator with generalized Calderón-Zygmund kernel $K$ if $T$ satisfies Assumption 12. We denote by $m-\operatorname{GCZK}(A, s, \eta, \epsilon)$ the set of functions $K$ satisfying (25), (66)-(67) with parameters $m, A, s, \eta$, and $\epsilon$. We also say $T$ is of class $m-\operatorname{GCZO}(A, s, \eta, \epsilon)$ if $T$ has an associated kernel $K$ in $m-\operatorname{GCZK}(A, s, \eta, \epsilon)$. 
Assumption 13. Assume that there exist operators $\left\{B_{t}\right\}_{t>0}$ with kernels $b_{t}(x, y)$ that satisfy conditions (66)-(67) with constants $s$ and $\eta$. Let

$$
K_{t}^{(0)}\left(x, y_{1}, \ldots, y_{m}\right)=\int_{\mathbb{R}^{n}} K\left(z, y_{1}, \ldots, y_{m}\right) b_{t}(x, z) d z,
$$

whenever $2 t^{1 / s} \leq \min _{1 \leq j \leq m}\left|x-y_{j}\right|$, and

$$
\begin{aligned}
\mid K & \left(x, y_{1}, \ldots, y_{m}\right)-K_{t}^{(0)}\left(x^{\prime}, y_{1}, \ldots, y_{m}\right) \mid \\
\leq & \frac{A}{\left(\sum_{k=1}^{m}\left|x-y_{k}\right|\right)^{m n}} \\
& \times \sum_{k=1, k \neq i}^{m} \phi\left(\frac{\left|y_{i}-y_{k}\right|}{t^{1 / s}}\right)+\frac{A t^{\epsilon / s}}{\left(\sum_{k=1}^{m}\left|x-y_{k}\right|\right)^{m n+\epsilon}},
\end{aligned}
$$

whenever $2\left|x-x^{\prime}\right| \leq t^{1 / s}$ and $2 t^{1 / s} \leq \max _{1 \leq j \leq m}\left|x-y_{i}\right|$.

It should be pointed out that the condition (67) is weaker than the condition (57) (see [23, Proposition 2.1]). Similarly, we can verify that Assumption 13 is weaker than the condition (57). These assumptions were introduced by Duong et al. in $[23,24]$. An important example for satisfying these assumptions is the $m$ th Calderón commutator. For $T$ in $m-\operatorname{GCZO}(A, s, \eta, \epsilon)$ with kernel $K$ satisfying Assumption 13 and the corresponding commutators $T_{\Sigma \vec{b}}$ and $T_{\Pi \vec{b}}$, lots of attention has been given (e.g., see [23-29] et al.). In particular, following from $[26,28]$, we have the following.

Lemma 14 (cf. [26, 28]). Assume that $T$ is a multilinear operator in $m-\operatorname{GCZO}(A, s, \eta, \epsilon)$ with kernel $K$ satisfying Assumption 13, $\vec{b}=\left(b_{1}, \ldots, b_{m}\right) \in B M O^{m}$. If there exist some $1 \leq q_{1}, \ldots, q_{m}<\infty$ and some $0<q<\infty$ with $1 / q=$ $\sum_{i=1}^{m} 1 / q_{i}$, such that $T$ maps $L^{q_{1}} \times \cdots \times L^{q_{m}}$ to $L^{q, \infty}$, then for $1 / m<p<\infty, 1<p_{1}, \ldots, p_{m}<\infty$ with $1 / p=\sum_{i=1}^{m} 1 / p_{i}$, $\vec{P}=\left(p_{1}, \ldots, p_{m}\right)$, and $\vec{w} \in A_{\vec{P}}$,

$$
\begin{aligned}
& \|T(\vec{f})\|_{L_{v_{\vec{w}}}^{p}\left(\mathbb{R}^{n}\right)} \leq C \prod_{j=1}^{m}\left\|f_{j}\right\|_{L_{w_{j}}^{p_{j}}\left(\mathbb{R}^{n}\right)^{\prime}} \\
& \left\|T_{\Sigma \vec{b}}(\vec{f})\right\|_{L_{v_{\vec{w}}}^{p}\left(\mathbb{R}^{n}\right)} \leq C\left(\sum_{j=1}^{m}\left\|b_{j}\right\|_{B M O\left(\mathbb{R}^{n}\right)}\right) \prod_{j=1}^{m}\left\|f_{j}\right\|_{L_{w_{j}}^{p_{j}}\left(\mathbb{R}^{n}\right)^{n}}, \\
& \left\|T_{\Pi \vec{b}}(\vec{f})\right\|_{L_{v_{\vec{w}}}^{p}\left(\mathbb{R}^{n}\right)} \leq C\left(\prod_{j=1}^{m}\left\|b_{j}\right\|_{B M O\left(\mathbb{R}^{n}\right)}\right) \prod_{j=1}^{m}\left\|f_{j}\right\|_{L_{w_{j}}^{p_{j}}\left(\mathbb{R}^{n}\right)^{\prime}},
\end{aligned}
$$

where $v_{\vec{w}}=\prod_{j=1}^{m} w_{j}^{p / p_{j}}$.

Invoking this lemma and Theorems 3-6, one has the following results.

Theorem 15. Let $m \in \mathbb{N}$ with $m \geq 2$ and $T$ be an $m$-linear operator in $m-G C Z O(A, s, \eta, \epsilon)$ with the kernel $K$ satisfying Assumption 13. Let $1<q_{j} \leq \alpha_{j}<p_{j}<\infty(j=1, \ldots, m)$ satisfy $1 / q=\sum_{i=1}^{m} 1 / q_{i}, 1 / \alpha=\sum_{i=1}^{m} 1 / \alpha_{i}, 1 / p=\sum_{i=1}^{m} 1 / p_{i}$, and $p / p_{j}=q / q_{j}=\alpha / \alpha_{j}(j=1, \ldots, m)$. Assume that $\vec{w}=\left(w_{1}, \ldots, w_{m}\right) \in \prod_{j=1}^{m} A_{q_{j}}, v_{\vec{w}}=\prod_{j=1}^{m} w_{j}^{q / q_{j}}$, and $\vec{b}=$ $\left(b_{1}, \ldots, b_{m}\right) \in B M O^{m}$. If there exist some $1 \leq r_{1}, \ldots, r_{m}<\infty$ and some $0<r<\infty$ with $1 / r=\sum_{i=1}^{m} 1 / r_{i}$, such that $T$ maps $L^{r_{1}}\left(\mathbb{R}^{n}\right) \times \cdots \times L^{r_{m}}\left(\mathbb{R}^{n}\right)$ to $L^{r, \infty}\left(\mathbb{R}^{n}\right)$, then

$$
\begin{aligned}
& \|T(\vec{f})\|_{\left(L_{v_{\vec{w}}}^{q}, L^{p}\right)^{\alpha}\left(\mathbb{R}^{n}\right)} \leq C \prod_{j=1}^{m}\left\|f_{j}\right\|_{\left(L_{w_{j}}^{q_{j}}, L^{p_{j}}\right)^{\alpha_{j}}\left(\mathbb{R}^{n}\right)}, \\
& \left\|T_{\Sigma \vec{b}}(\vec{f})\right\|_{\left(L_{v_{\vec{w}}}^{q}, L^{p}\right)^{\alpha}\left(\mathbb{R}^{n}\right)} \\
& \leq C\left(\sum_{j=1}^{m}\left\|b_{j}\right\|_{B M O\left(\mathbb{R}^{n}\right)}\right) \prod_{j=1}^{m}\left\|f_{j}\right\|_{\left(L_{w_{j}}^{\left.q_{j}, L^{p_{j}}\right)}\right)^{\alpha_{j}}\left(\mathbb{R}^{n}\right)^{\prime}} \\
& \left\|T_{\Pi \vec{b}}(\vec{f})\right\|_{\left(L_{v_{\vec{w}}}^{q}, L^{p}\right)^{\alpha}\left(\mathbb{R}^{n}\right)} \\
& \leq C\left(\prod_{j=1}^{m}\left\|b_{j}\right\|_{B M O\left(\mathbb{R}^{n}\right)}\right) \prod_{j=1}^{m}\left\|f_{j}\right\|_{\left(L_{w_{j}}^{q_{j}} L^{p_{j}}\right)^{\alpha_{j}}\left(\mathbb{R}^{n}\right)},
\end{aligned}
$$

where $C$ depends only on $m, n, p, q, \alpha$ and $[w]_{A_{q_{j}}}$.

In particular, by Remark 7 again and Lemma 14, one has the following.

Theorem 16. Let $m \in \mathbb{N}$ with $m \geq 2$ and $T$ be an $m$-linear operator in $m-G C Z O(A, s, \eta, \epsilon)$ with the kernel $K$ satisfying Assumption 13. Let $1<q_{j} \leq \alpha_{j}<p_{j}<\infty(j=1, \ldots, m)$ satisfy $1 / q=\sum_{i=1}^{m} 1 / q_{i}, 1 / \alpha=\sum_{i=1}^{m} 1 / \alpha_{i}$, and $1 / p=\sum_{i=1}^{m} 1 / p_{i}$. Assume that $w \in A_{q}, \vec{b}=\left(b_{1}, \ldots, b_{m}\right) \in B M O^{m}$. If there exist some $1 \leq r_{1}, \ldots, r_{m}<\infty$ and some $0<r<\infty$ with $1 / r=\sum_{i=1}^{m} 1 / r_{i}$, such that $T$ maps $L^{r_{1}}\left(\mathbb{R}^{n}\right) \times \cdots \times L^{r_{m}}\left(\mathbb{R}^{n}\right)$ to $L^{r, \infty}\left(\mathbb{R}^{n}\right)$, then

$$
\|T(\vec{f})\|_{\left(L_{w}^{q}, L^{p}\right)^{\alpha}\left(\mathbb{R}^{n}\right)} \leq C \prod_{j=1}^{m}\left\|f_{j}\right\|_{\left(L_{w}^{q_{j}}, L^{p_{j}}\right)^{\alpha_{j}}\left(\mathbb{R}^{n}\right)^{\prime}}
$$

$$
\begin{aligned}
& \left\|T_{\Sigma \vec{b}}(\vec{f})\right\|_{\left(L_{w}^{q}, L^{p}\right)^{\alpha}\left(\mathbb{R}^{n}\right)} \\
& \quad \leq C\left(\sum_{j=1}^{m}\left\|b_{j}\right\|_{B M O\left(\mathbb{R}^{n}\right)}\right) \prod_{j=1}^{m}\left\|f_{j}\right\|_{\left(L_{w}^{q_{j}}, L^{p_{j}}\right)^{\alpha_{j}}\left(\mathbb{R}^{n}\right)},
\end{aligned}
$$

$$
\begin{aligned}
& \left\|T_{\Pi \vec{b}}(\vec{f})\right\|_{\left(L_{w}^{q}, L^{p}\right)^{\alpha}\left(\mathbb{R}^{n}\right)} \\
& \quad \leq C\left(\prod_{j=1}^{m}\left\|b_{j}\right\|_{B M O\left(\mathbb{R}^{n}\right)}\right) \prod_{j=1}^{m}\left\|f_{j}\right\|_{\left(L_{w}^{q_{j}}, L^{p_{j}}\right)^{\alpha_{j}}\left(\mathbb{R}^{n}\right)^{\prime}}
\end{aligned}
$$

where $C$ depends only on $m, n, p, q, \alpha$ and $[w]_{A_{q}}$.

\section{Conflict of Interests}

The authors declare that there is no conflict of interests regarding the publication of this paper. 


\section{Acknowledgment}

This work was supported by the NNSF of China (nos. $11071200,11371295)$.

\section{References}

[1] I. Fofana, "Continuité de l'intégrale fractionnaire et espace $\left(L^{q}, l^{p}\right)^{\alpha}$," Comptes Rendus des Séances de l'Académie des Sciences $I$, vol. 308, no. 18, pp. 525-527, 1989.

[2] Y. Komori and S. Shirai, "Weighted Morrey spaces and a singular integral operator," Mathematische Nachrichten, vol. 282, no. 2, pp. 219-231, 2009.

[3] T. Iida, E. Sato, Y. Sawano, and H. Tanaka, "Multilinear fractional integrals on Morrey spaces," Acta Mathematica Sinica (English Series), vol. 28, no. 7, pp. 1375-1384, 2012.

[4] L. Liu, "Boundedness for multilinear singular integral operators on Morrey spaces," Bulletin of the Malaysian Mathematical Sciences Society II, vol. 33, no. 1, pp. 93-103, 2010.

[5] Z. Si, "Commutator theorems for fractional integral operators on Morrey spaces," http://arxiv.org/abs/1203.4407.

[6] H. Wang and W. Yi, "Multilinear singular and fractional integral operators on weighted Morrey spaces," Journal of Function Spaces and Applications, vol. 2013, Article ID 735795, 11 pages, 2013.

[7] A. Bonami, J. Feuto, and I. Fofana, "Norms inequalities in some subspaces of Morrey space," http://arxiv.org/abs/1211.3565.

[8] J. Feuto, "Norm inequalities in a class of function spaces including weighted Morrey spaces," http://arxiv.org/abs/1205.6516.

[9] J. Feuto, "Intrinsic square functions on functions spaces including weighted Morrey spaces," http://arxiv.org/abs/1205.0129.

[10] J. Feuto, I. Fofana, and K. Koua, "Weighted norm inequalities for a maximal operator in some subspace of amalgams," Canadian Mathematical Bulletin, vol. 53, no. 2, pp. 263-277, 2010.

[11] A. K. Lerner, S. Ombrosi, C. Pérez, R. H. Torres, and R. TrujilloGonzález, "New maximal functions and multiple weights for the multilinear Calderón-Zygmund theory," Advances in Mathematics, vol. 220, no. 4, pp. 1222-1264, 2009.

[12] T. Iida, E. Sato, Y. Sawano, and H. Tanaka, "Sharp bounds for multilinear fractional integral operators on Morrey type spaces," Positivity, vol. 16, no. 2, pp. 339-358, 2012.

[13] R. R. Coifman and Y. Meyer, "On commutators of singular integrals and bilinear singular integrals," Transactions of the American Mathematical Society, vol. 212, pp. 315-331, 1975.

[14] R. R. Coifman and Y. Meyer, "Commutateurs d'intégrales singulières et opérateurs multilinéaires," Annales de l'Institut Fourier, vol. 28, no. 3, pp. 177-202, 1978.

[15] M. Christ and J. L. Journé, "Polynomial growth estimates for multilinear singular integral operators," Acta Mathematica, vol. 159, no. 1-2, pp. 51-80, 1987.

[16] C. E. Kenig and E. M. Stein, "Multilinear estimates and fractional integration," Mathematical Research Letters, vol. 6, no. 1, pp. 1-15, 1999.

[17] L. Grafakos and R. H. Torres, "Multilinear Calderón-Zygmund theory," Advances in Mathematics, vol. 165, no. 1, pp. 124-164, 2002.

[18] L. Grafakos and R. H. Torres, "Maximal operator and weighted norm inequalities for multilinear singular integrals," Indiana University Mathematics Journal, vol. 51, no. 5, pp. 1261-1276, 2002.
[19] L. Tang, "Weighted estimates for vector-valued commutators of multilinear operators," Proceedings of the Royal Society of Edinburgh A, vol. 138, no. 4, pp. 897-922, 2008.

[20] C. Pérez, G. Pradolini, R. H. Torres, and R. Trujillo-Gonzalez, "End-point estimates for iterated commutators of multilinear singular integrals," Bulletin of the London Mathematical Society, vol. 46, no. 1, pp. 26-42, 2014.

[21] C. Pérez and R. H. Torres, "Sharp maixmal function estimates for multilinear singular integrals," Contemporary Mathematics, vol. 320, pp. 323-331, 2003.

[22] X. T. Duong and A. MacIntosh, "Singular integral operators with non-smooth kernels on irregular domains," Revista Matemática Iberoamericana, vol. 15, no. 2, pp. 233-265, 1999.

[23] X. T. Duong, L. Grafakos, and L. Yan, "Multilinear operators with non-smooth kernels and commutators of singular integrals," Transactions of the American Mathematical Society, vol. 362, no. 4, pp. 2089-2113, 2010.

[24] X. T. Duong, R. Gong, L. Grafakos, J. Li, and L. Yan, "Maximal operator for multilinear singular integrals with non-smooth kernels," Indiana University Mathematics Journal, vol. 58, no. 6, pp. 2517-2541, 2009.

[25] B. T. Anh and X. T. Duong, "On commutators of vector BMO functions and multilinear singular integrals with non-smooth kernels," Journal of Mathematical Analysis and Applications, vol. 371, no. 1, pp. 80-94, 2010.

[26] S. Chen and H. Wu, "Multiple weighted estimates for commutators of multilinear singular integrals with non-smooth kernels," Journal of Mathematical Analysis and Applications, vol. 396, no. 2, pp. 888-903, 2012.

[27] R. M. Gong and J. Li, "Sharp maximal function estimates for multilinear singular integrals with non-smooth kernels," Analysis in Theory and Applications, vol. 25, no. 4, pp. 333-348, 2009.

[28] L. Grafakos, L. Liu, and D. Yang, "Multiple-weighted norm inequalities for maximal multi-linear singular integrals with non-smooth kernels," Proceedings of the Royal Society of Edinburgh A, vol. 141, no. 4, pp. 755-775, 2011.

[29] J. Lian, J. Li, and H. Wu, "Multilinear commutators of BMO functions and multilinear singular integrals with non-smooth kernels," Applied Mathematics: A Journal of Chinese Universities, vol. 26, no. 1, pp. 109-120, 2011. 


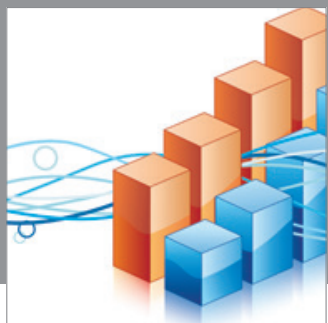

Advances in

Operations Research

mansans

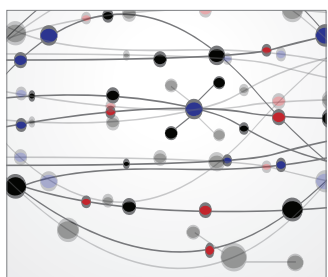

The Scientific World Journal
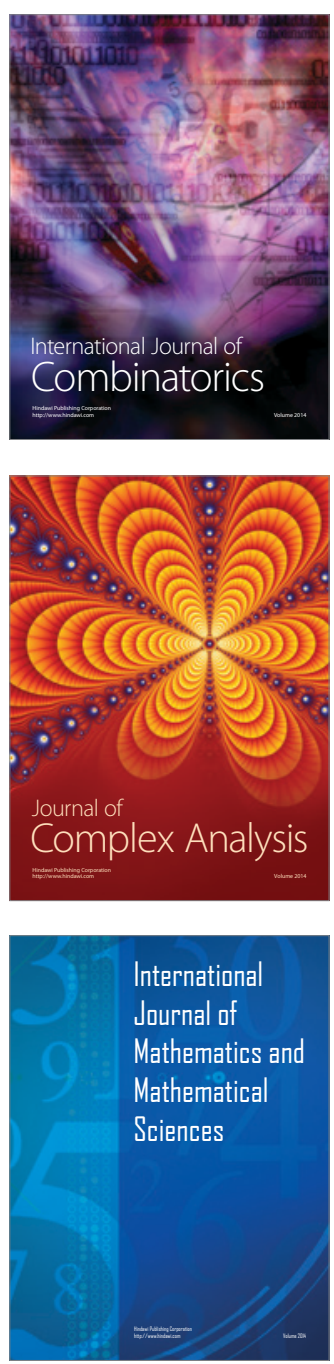
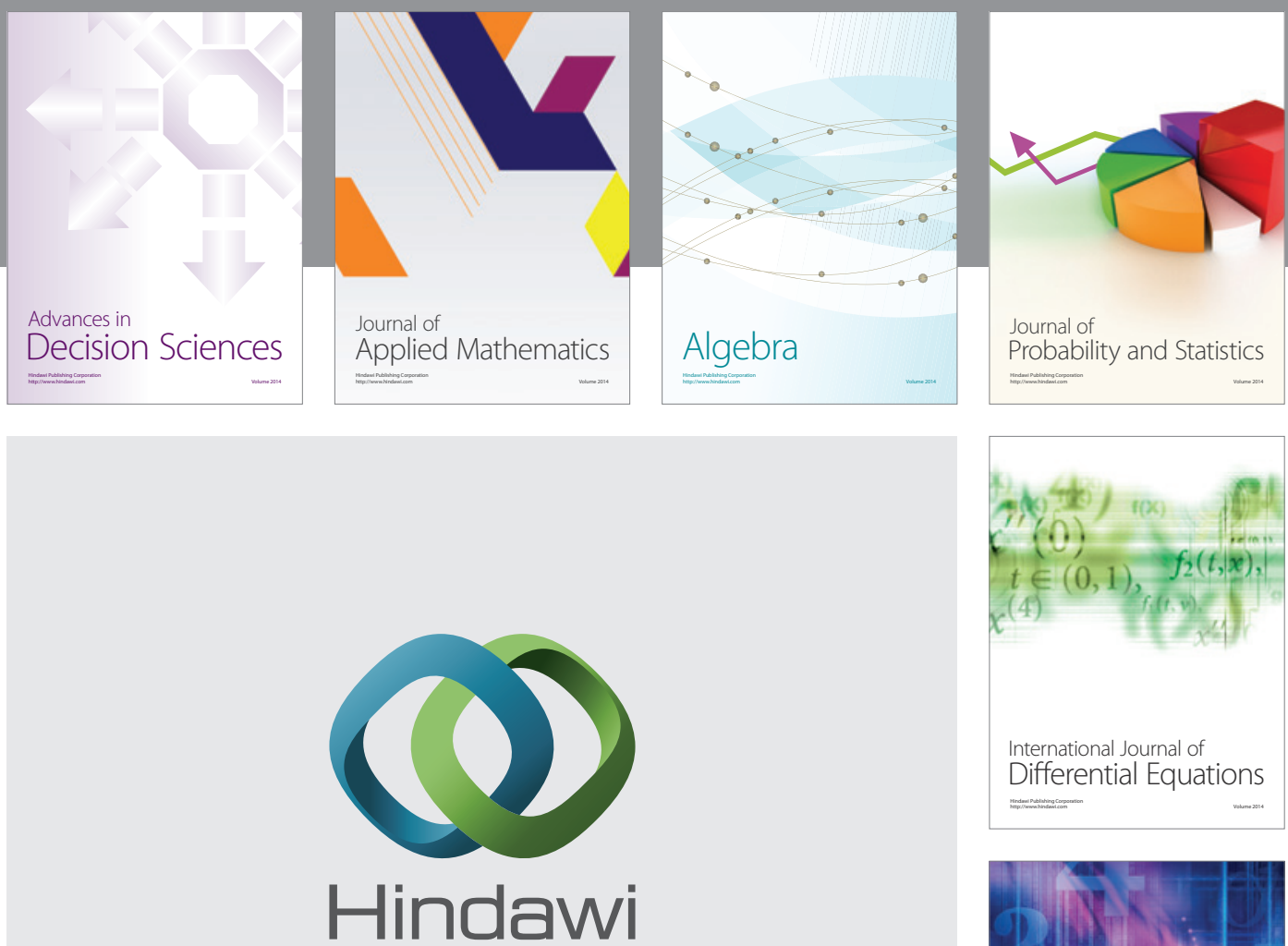

Submit your manuscripts at http://www.hindawi.com
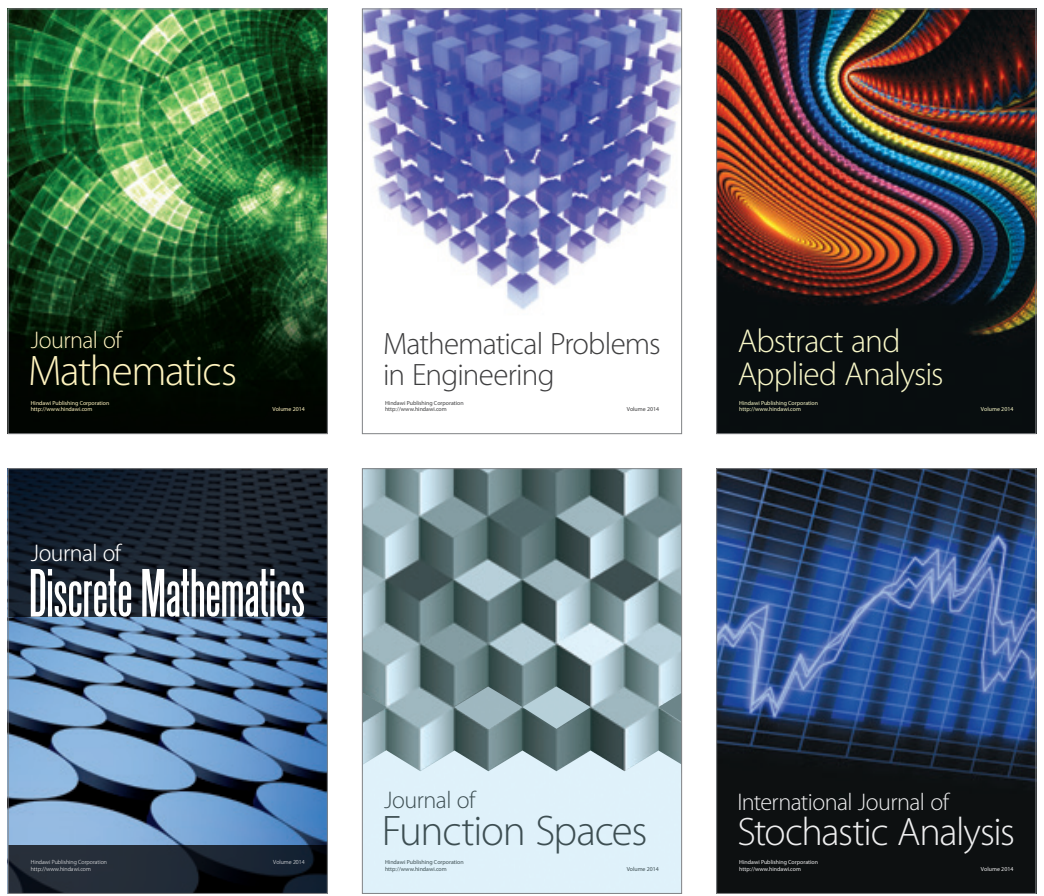

Journal of

Function Spaces

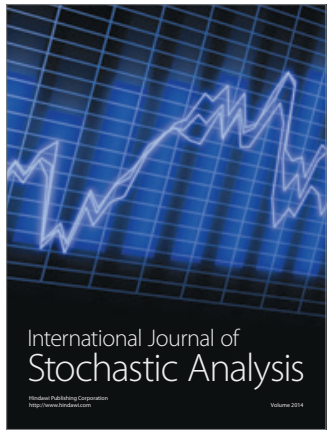

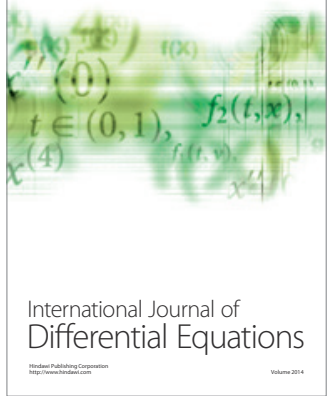
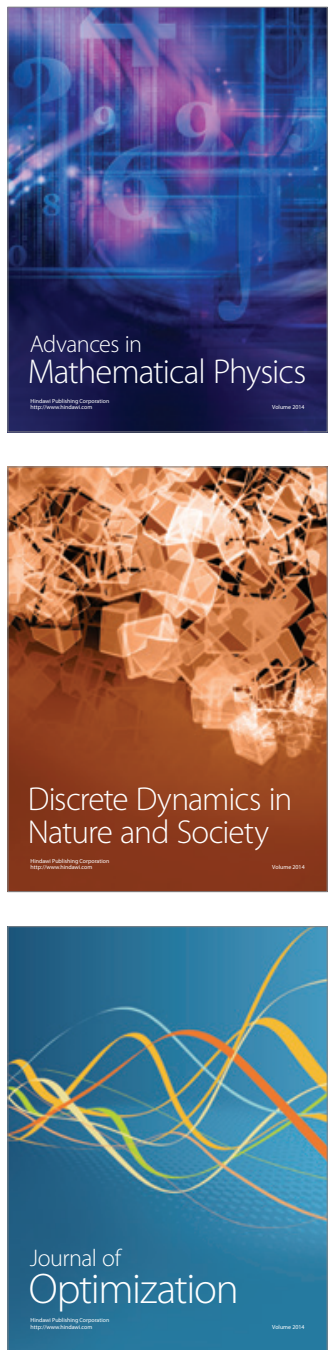